\title{
Saddle solutions for bistable symmetric semilinear elliptic equations
}

\author{
Francesca Alessio and Piero Montecchiari
}

\begin{abstract}
This paper concerns the existence and asymptotic characterization of saddle solutions in $\mathbb{R}^{3}$ for semilinear elliptic equations of the form

$$
-\Delta u+W^{\prime}(u)=0, \quad(x, y, z) \in \mathbb{R}^{3}
$$

where $W \in \mathcal{C}^{3}(\mathbb{R})$ is a double well symmetric potential, i.e. it satisfies $W(-s)=W(s)$ for $s \in \mathbb{R}, W(s)>0$ for $s \in(-1,1), W( \pm 1)=0$ and $W^{\prime \prime}( \pm 1)>0$. Denoted with $\theta_{2}$ the saddle planar solution of $(0.1)$, we show the existence of a unique solution $\theta_{3} \in C^{2}\left(\mathbb{R}^{3}\right)$ which is odd with respect to each variable, symmetric with respect to the diagonal planes, verifies $0<\theta_{3}(x, y, z)<1$ for $x, y, z>0$ and $\theta_{3}(x, y, z) \rightarrow z \rightarrow+\infty \theta_{2}(x, y)$ uniformly with respect to $(x, y) \in \mathbb{R}^{2}$.
\end{abstract}

Mathematics Subject Classification (2010). 35J60, 35B05, 35B40, 35J20, 34C37.

Keywords. Elliptic equations, Variational methods, Entire solutions.

\section{Introduction}

We consider on $\mathbb{R}^{3}$, semilinear elliptic equations of the form

$$
-\Delta u+W^{\prime}(u)=0
$$

assuming that $W$ is a double well symmetric potential, i.e.

$W \in \mathcal{C}^{3}(\mathbb{R})$ satisfies $W(-s)=W(s)$ for $s \in \mathbb{R}, W(s)>0$ for $s \in(-1,1)$,

$W( \pm 1)=0$ and $W^{\prime \prime}( \pm 1)>0$.

The problem of looking for and classifying entire bounded solutions of this kind of equations in $\mathbb{R}^{n}$ has been extensively studied in the last years, providing a rich amount of differently shaped families of solutions. In particular, a

This work was partially supported by the PRIN2009 grant "Critical Point Theory and Perturbative Methods for Nonlinear Differential Equations". 
long standing problem pointed out by De Giorgi in [16], is to characterize the set of the solutions $u \in C^{2}\left(\mathbb{R}^{n}\right)$ of $(1.1)$, satisfying $|u(x)| \leq 1$ and $\partial_{x_{1}} u(x)>0$ in $\mathbb{R}^{n}$. In [16], De Giorgi conjectured that if $n \leq 8$ and $W(s)=\left(s^{2}-1\right)^{2}$, any solutions of (1.1) of that kind, coincides up to space roto-translations with the unique solution $\theta_{1}$ of the one dimensional heteroclinic problem

$$
\left\{\begin{array}{l}
-\ddot{q}(x)+W^{\prime}(q(x))=0, \quad x \in \mathbb{R} \\
q(0)=0 \text { and } q( \pm \infty)= \pm 1,
\end{array}\right.
$$

The conjecture has been proved for $n=2$ by Ghoussoub and Gui in [29] and then by Ambrosio and Cabrè in [8] (see also [2]) for $n=3$, even for more general double well potentials $W$. A further step in the proof of the De Giorgi conjecture has been done by Savin in [39] where, when $n \leq 8$, the same one dimensional symmetry is obtained for solutions $u$ such that $\partial_{x_{1}} u(x)>0$ on $\mathbb{R}^{n}$ and $\lim _{x_{1} \rightarrow \pm \infty} u(x)= \pm 1$ for all $\left(x_{2}, x_{3}, \ldots, x_{n}\right) \in \mathbb{R}^{n-1}$ (see $[9,11,24-27,40]$ for related problems). That result is completed in $[17,18]$ where the existence of entire solutions without one dimensional symmetry which are increasing and asymptotic to \pm 1 with respect to the first variable is proved in dimension $n>8$.

Non monotonic solutions of equation (1.1) are the saddle solutions, introduced by Dang et al. in [15]. In that paper the Authors consider potentials $W$ satisfying $(W)$ togheter with the condition

$W^{\prime \prime}(0)<0$ and the function $u \rightarrow W^{\prime}(u) / u$ is strictly increasing on $(0,1)$.

Under these assumptions They prove that (1.1) has a unique solution $u \in$ $C^{2}\left(\mathbb{R}^{2}\right)$ which is odd w.r.t. $x$ and $y$ and which is positive when $x y>0$. In particular the saddle solution $u$ has the same sign of the function $x y$. The monotonicity of the function $W^{\prime}(u) / u$ allows the Authors to prove their result by the use of a supersolution-subsolution method.

Assuming $(W)-(W 1)$, Schatzman in [41] studied the stability properties of the saddle solution proving that its Morse index is at least equal to one and, more recently, Kowalczyk and Liu in [33] proved that the saddle solution is not degenerate in the sense that the corresponding linearized operator has zero kernel in $L^{2}\left(\mathbb{R}^{2}\right)$. Cabré and Terra in $[13,14]$ found in all even dimensions saddle type solutions (vanishing on the Simons' cones) characterizing their instability for dimensions $n=4,6$. A vectorial version of the result in [15] has been obtained by Alama et al. in [1] where systems of equations of the type (1.1) have been studied. We refer also to [30] where, among other results, existence, uniqueness and qualitative properties of the saddle solution of (1.1) has been studied by Gui assuming $(W)$ and

$$
W^{\prime}(u) \neq 0 \quad \text { for } u \in(0,1) .
$$

The saddle solutions are examples of four ends solutions, which, roughly speaking, are planar solutions characterized by the fact that their nodal set is asymptotic at infinity to four half lines. This kind of solutions of (1.1) has been studied assuming $(W)$ and $(W 2)$ in $[20,31,34,35]$ (see also [19]). In particular, 
in [31] Gui proved that any four ends solution is, modulo roto-translations, even in its variables and monotonic in $x$ and $y$ on the first quadrant. In [35] it is shown that the set of even four end solutions constitutes a continuum containing the saddle solution.

The existence of more general $2 \mathrm{k}$ ends solutions with nearly parallel ends has been obtained in [20] for every $k \in \mathbb{N}$ in the case of the Allen Cahn potential. Other $2 \mathrm{k}$ ends solutions, built in [3] for potential satisfying $(W)$, are saddle type solutions with nodal set equal to the union of $k$ lines through the origin forming angles equal to $\pi / k$.

All these solutions are characterized by the fact that along directions parallel to the end lines they are asymptotic to the one dimensional solution $\theta_{1}$ (i.e. the saddle solution in dimension 1), suitably reflected and rotated, and along different directions they tend to \pm 1 in an alternate way with respect to the end lines. In connection with the De Giorgi conjecture we remark that if $k>1$ is odd, up to a rotation, the $2 \mathrm{k}$ ends solutions are example of non monotonic planar solution $u(x, y)$ of (1.1) which satisfy the asymptotic condition $\lim _{x \rightarrow \pm \infty} u(x, y)= \pm 1$ for every $y \in \mathbb{R}$.

In the present work we introduce a global variational approach suitable to study under the assumption $(W)$ the problem of existence of a saddle type solutions of (1.1) in $\mathbb{R}^{3}$ providing directly a characterization of its asymptotic behaviour. Indeed, as showed in [3] and recalled in Sect. 3.1 below, the assumption $(W)$ is sufficient to obtain existence and uniqueness of the planar saddle solution of (1.1) which we denote $\theta_{2}(x, y)$. The main Theorem of the paper is the following one

Theorem 1.1. Assuming $(W)$ there exists a unique solution $\theta_{3} \in C^{2}\left(\mathbb{R}^{3}\right)$ of (1.1) verifying

i) $\left|\theta_{3}(x, y, z)\right|<1$ on $\mathbb{R}^{3}$ and $\theta_{3}(x, y, z)>0$ for $x, y, z>0$,

ii) $\theta_{3}$ is odd with respect to each variable and symmetric with respect to the diagonal planes $x=y, x=z$ and $y=z$,

iii) $\theta_{3}(x, y, z) \rightarrow \theta_{2}(x, y)$ as $z \rightarrow+\infty$ uniformly with respect to $(x, y) \in \mathbb{R}^{2}$.

Moreover $\theta_{3}(x, y, z)$ is increasing in each variable on the set $\{(x, y, z) /$ $x, y, z>0\}$.

We note that, since $\theta_{3}$ is odd with respect to each of its variable and strictly positive on the set $\{(x, y, z) / x, y, z>0\}$, there results that $\theta_{3}$ has the same sign of the product function $x y z$ and its nodal set coincides with the union of the three cartesian planes. We remark that the mere existence of solutions of that kind could be proved in $\mathbb{R}^{n}$ also for $n \geq 3$, at least in the case in which also $(W 2)$ holds true, by using a suitable direct minimization argument on balls of radius $R$ and then considering the limit as $R$ goes to infinity.

The more tricky and interesting result in Theorem 1.1 is the asymptotic characterization of $\theta_{3}$ given by property (iii) (see [32] for related results in the vectorial setting). Thanks to the symmetry properties of $\theta_{3}$, (iii) tells us, analogously to the planar case, that along directions parallel to the axes, $\theta_{3}$ is uniformly asymptotic to the two dimensional saddle solution $\theta_{2}$, suitably 
reflected and rotated. In this line one can try to generalize Theorem 1.1 in different ways. For example, analogous to the $k$-end planar solutions, one can try to find entire solutions on $\mathbb{R}^{3}$ which along different directions are asymptotic to $\theta_{2}$ (reflected and rotated), or, one can ask whether an inductive statement of the same type holds true in any dimension: if $\theta_{n-1}$ is a saddle solution in $\mathbb{R}^{n-1}$ then there exists a unique $\theta_{n}$ satisfying (i) and (ii) in $\mathbb{R}^{n}$ and such that $\theta_{n}\left(x_{1}, \ldots, x_{n}\right) \rightarrow_{x_{n} \rightarrow+\infty} \theta_{n-1}\left(x_{1}, \ldots, x_{n-1}\right)$ for $\left(x_{1}, \ldots, x_{n-1}\right) \in \mathbb{R}^{n-1}$.

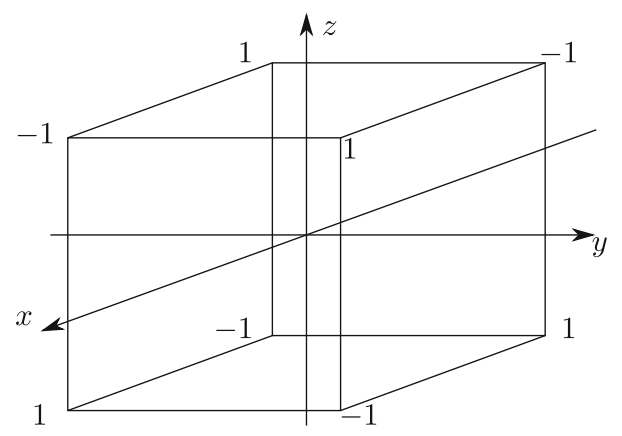

We remark also that, thanks to $($ iii $), \theta_{3}$ is asymptotic to \pm 1 along directions parallel to the space diagonals and so, again in connection with the De Giorgi conjecture, rotating the saddle solution $\theta_{3}$ we find an example in $\mathbb{R}^{3}$ of non monotonic entire solution $u(x, y, z)$ of (1.1) which satisfy the asymptotic condition $\lim _{x \rightarrow \pm \infty} u(x, y, z)= \pm 1$ for every $(y, z) \in \mathbb{R}^{2}$.

A last comment regards the uniqueness of $\theta_{3}$ which is obviously linked again to property $(i i i)$. Indeed we note that since only $(W)$ is assumed, up to dilation and the addition of a constant to $W$, Theorem 1.1 applies to general even potential $W \in C^{3}(R)$ having a local non degenerate minimum point $a>0$ for which $W(s)>W(a)$ for $s \in(-a, a)$. In the case in which $W$ has different non degenerate positive minimum points $a_{i}>0$ satisfying that property, Theorem 1.1 gives different solutions $\theta_{3, i}$ of (1.1), all odd with respect to each variable and positive on the set $\{(x, y, z) / x, y, z>0\}$, but clearly characterized by different asymptotic behaviours.

To prove Theorem 1.1 we develop a global variational procedure, inspired to the ones introduced in $[3,7]$, which allows us to recover the saddle type solution $\theta_{3}$ from the minimum of a suitably renormalized action functional (for the use of renormalized functionals in different contexts we also refer to $[4-6,36]$ and to the comprehensive recent monograph [37]). We look for minima of the double renormalized functional

$F_{\infty, 3}(u)=\int_{0}^{+\infty}\left[\int_{0}^{z}\left[\int_{-y}^{y} \frac{1}{2}|\nabla u(x, y, z)|^{2}+a(x) W(u(x, y, z)) d x-c_{1, y}\right] d y-c_{2, z}\right] d z$

on the class $\Gamma_{\infty, 3}=\left\{u \in H_{l o c}^{1}\left(T_{\infty, 3}\right) / u(x, y, z)=-u(-x, y, z)\right.$ and $0 \leq$ $u(x, y, z) \leq 1$ for $x>0\}$, where $T_{\infty, 3}=\left\{(x, y, z) \in \mathbb{R}^{3} / 0<|x|<y<z\right\}$. In the definition of $F_{\infty, 3}$ enter the two renormalizing functions $c_{1, y}$ and $c_{2, z}$ which are related to minimal problems in dimension 1 and 2 . Precisely 


$$
c_{1, y}=\min \left\{\int_{-y}^{y} \frac{1}{2}|\dot{q}|^{2}+W(q) d x / q \in \Gamma_{y, 1}\right\}
$$

where $\Gamma_{y, 1}=\left\{q \in H^{1}(-y, y) / q(x)=-q(-x), 0 \leq q(x) \leq 1\right.$ for $\left.x>0\right\}$, and

$$
\left.c_{2, z}=\min \left\{\int_{0}^{z} \int_{-y}^{y} \frac{1}{2}|\nabla u|^{2}+W(u) d x-c_{1, y} d y / u \in \Gamma_{z, 2}\right)\right\}
$$

where $\Gamma_{z, 2}=\left\{u \in H^{1}\left(T_{z, 2}\right) / u(x, y)=-u(-x, y), 0 \leq u(x, y) \leq 1\right.$ for $\left.x>0\right\}$ and $T_{z, 2}=\left\{(x, y) \in \mathbb{R}^{2} / 0<|x|<y<z\right\}$.

This global variational approach allows us to directly control the asymptotes as $z \rightarrow+\infty$ of the minima of $F_{\infty, 3}$. Indeed, if $u$ is a minimum, then $\sup _{(x, y) \in T_{2, z}}\left|v(x, y, z)-\theta_{2}(x, y)\right| \rightarrow 0$ as $z \rightarrow+\infty$ (see Lemmas 4.2 and 4.3 below). Moreover a minimum of $F_{\infty, 3}$ is in fact minimal with respect to $C_{0}^{\infty}\left(\mathbb{R}^{3}\right)$ perturbation (see Lemma 4.1 below) and the saddle solution $\theta_{3}$ is recovered from it by recursive reflections with respect to the faces of $T_{\infty, 3}$. The uniqueness and the monotonicity properties of $\theta_{3}$ are obtained by standard elliptic techniques.

We remark that, even if obtained by recursive reflections of a minimal solution, the solution $\theta_{3}$ is no more minimal for the action with respect to $\mathcal{C}_{0}^{\infty}\left(\mathbb{R}^{3}\right)$ perturbations. In fact, since finite Morse index solutions are necessarily stable at infinity and since $\theta_{2}$ has Morse index at least equal to 1 (see [41]), $\theta_{3}$ has infinite Morse index. For finite Morse index solutions in $\mathbb{R}^{3}$ we refer to the papers by Del Pino et al. [22,23], where solutions concentrating around complete embedded minimal surfaces of finite total curvature are constructed.

The paper is organized as follows. Sections 2 and 3 are devoted to recall and study a list of refined properties of the one dimensional and two dimensional problems, characterizing in particular the asymptotic properties of the minimal functions $c_{1, y}$ and $c_{2, z}$. The double renormalized functional $F_{\infty, 3}$ is defined and studied in Sect. 4 , where we complete the proof of Theorem 1.1.

Remark 1.1. We precise now some basic consequences of the assumption $(W)$ fixing some constants which will remain unchanged in the rest of the paper. First, since $W \in \mathcal{C}^{3}(\mathbb{R})$ and $W^{\prime \prime}( \pm 1)>0$, there exists $\bar{\delta} \in\left(0, \frac{1}{4}\right)$ such that setting $\underline{w}=W^{\prime \prime}( \pm 1) / 2$ and $\bar{w}=3 \underline{w}$ we have

$$
\bar{w} \geq W^{\prime \prime}(s) \geq \underline{w} \quad \text { for any }|s| \in[1-2 \bar{\delta}, 1+2 \bar{\delta}] .
$$

In particular, since $W( \pm 1)=W^{\prime}( \pm 1)=0$, setting $\chi(s)=\min \{|1-s|,|1+s|\}$, we have that

$$
\frac{\underline{w}}{2} \chi(s)^{2} \leq W(s) \leq \frac{\bar{w}}{2} \chi(s)^{2} \quad \text { and } \quad\left|W^{\prime}(s)\right| \leq \bar{w} \chi(s), \forall|s| \in[1-2 \bar{\delta}, 1+2 \bar{\delta}]
$$

Finally, since we are looking for solution $u$ such that $|u(x, y, z)| \leq 1$, by $(W)$ it is not restrictive to assume, and in fact we will do it below, that $W(s) \geq 0$ for $s \in \mathbb{R}$. 


\section{The one dimensional problem}

In this section we describe some results concerning the one dimensional ODE associated to (1.1).

\subsection{The heteroclinic problem.}

We consider the heteroclinic ODE problem associated to (1.1)

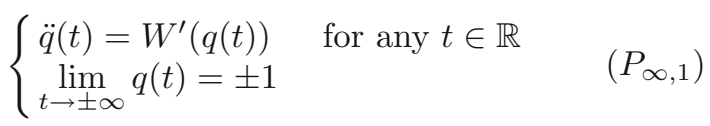

It is well known, that the problem admits a unique solution modulo time translation and we denote $\theta_{1}$ the solution which satisfies $\theta_{1}(0)=0$. We recall here below some relevant and khow properties of the solution $\theta_{1}$.

Remark 2.1. (Symmetry, monotonicity and exponential decaying of $\theta_{1}$ ). The function $\theta_{1}$ is odd and increasing:

$$
\theta_{1}(x)=-\theta_{1}(-x) \quad \text { and } \quad \dot{\theta}_{1}(x)>0 \quad \text { for every } x \in \mathbb{R} .
$$

In particular $\left|\theta_{1}(x)\right|<1$. Moreover $\theta_{1}$ behaves exponentially at infinity verifying that there exists a constant $C>0$ such that

$$
0 \leq 1-\theta_{1}(x) \leq C \mathrm{e}^{-\sqrt{\frac{w}{2}} x} \text { and } 0<\dot{\theta}_{1}(x) \leq C \mathrm{e}^{-\sqrt{\frac{w}{2}} x} \text { for all } x>0 .
$$

Remark 2.2. (Non Degeneracy property of $\theta_{1}$ ) The set of bounded solutions of the linearized equation about $\theta_{1},-\ddot{h}(x)+W^{\prime \prime}\left(\theta_{1}(x)\right) h(x)=0, x \in \mathbb{R}$, is one dimensional and contains the function $\dot{\theta}_{1}$.

Remark 2.3. (Minimality of $\theta_{1}$ ). We consider the action functional

$$
F_{\infty, 1}(q)=\int_{\mathbb{R}} \frac{1}{2}|\dot{q}(x)|^{2}+W(q(x)) d x .
$$

on the space

$$
\mathcal{H}=z_{0}+H^{1}(\mathbb{R}),
$$

where $z_{0} \in C^{\infty}(\mathbb{R})$ is a fixed odd increasing function such that $z_{0}(x) \rightarrow \pm 1$, as $x \rightarrow \pm \infty$, and $\left|z_{0}(x)\right|=1$ for any $|x| \geq 1$. Setting

$$
c_{\infty, 1}=\inf _{\mathcal{H}} F_{\infty, 1}
$$

we have $\theta_{1} \in \mathcal{H}$ and $F_{\infty, 1}\left(\theta_{1}\right)=c_{\infty, 1}$, i.e., $\theta_{1}$ is a minimum of $F_{\infty, 1}$ on $\mathcal{H}$.

Note that $\mathcal{H}$ is an affine Hilbertian Manifold if endowed with the $H^{1}(\mathbb{R})$ scalar product and $F \in C^{2}(\mathcal{H})$ with

$$
\begin{aligned}
F_{\infty, 1}^{\prime}\left(\theta_{1}\right) h & =\int_{\mathbb{R}} \dot{\theta}_{1} \dot{h}+W^{\prime}\left(\theta_{1}\right) h d x \quad \text { and } \\
F_{\infty, 1}^{\prime \prime}\left(\theta_{1}\right) h k & =\int_{\mathbb{R}} \dot{h} \dot{k}+W^{\prime \prime}\left(\theta_{1}\right) h k d x \quad \text { for any } h, k \in H^{1}(\mathbb{R}) .
\end{aligned}
$$

Since $\theta_{1}$ is a minimum of $F_{\infty, 1}$ on $\mathcal{H}$, we have in particular that $F_{\infty, 1}^{\prime}\left(\theta_{1}\right)=0$ and that $F_{\infty, 1}^{\prime \prime}\left(\theta_{1}\right) h h \geq 0$ for any $h \in H^{1}(\mathbb{R})$. 
Lemma 2.1. (Stability of $\left.\theta_{1}\right)$. There exists $\lambda_{1} \in(0,2 \underline{w}]$ such that for all odd functions $h \in H^{1}(\mathbb{R})$ there results

$$
F_{\infty, 1}^{\prime \prime}\left(\theta_{1}\right) h h=\int_{\mathbb{R}}|\dot{h}|^{2}+W^{\prime \prime}\left(\theta_{1}\right) h h d x \geq \lambda_{1}\|h\|_{L^{2}(\mathbb{R})}^{2} .
$$

Proof. Indeed, recalling that $2 \underline{w}=W^{\prime \prime}( \pm 1)$, since $\theta_{1}(x) \rightarrow \pm 1$ as $x \rightarrow \pm \infty$, we recover that the selfadjoint operator $\mathcal{L}_{\infty, 1}: H^{2}(\mathbb{R}) \subset L^{2}(\mathbb{R}) \rightarrow L^{2}(\mathbb{R})$,

$$
\mathcal{L}_{\infty, 1}(h)=-\ddot{h}+W^{\prime \prime}\left(\theta_{1}\right) h=-\Delta h+2 \underline{w} h+\left(W^{\prime \prime}\left(\theta_{1}\right)-2 \underline{w}\right) h
$$

is a compact perturbation of the operator $\AA: H^{2}(\mathbb{R}) \subset L^{2}(\mathbb{R}) \rightarrow L^{2}(\mathbb{R})$, $\stackrel{\circ}{h}=-\ddot{h}+2 \underline{w} h$. Then, the essential spectrum of $\mathcal{L}_{\infty, 1}$ coincides with the one of $\AA$, i.e., $\sigma_{\text {ess }}\left(\mathcal{L}_{\infty, 1}\right)=\sigma_{\text {ess }}(\AA)=[2 \underline{w},+\infty)$. By the minimality property of $\theta_{1}$ (Remark 2.3) we know that $\left\langle\mathcal{L}_{\infty, 1}(h), h\right\rangle_{L^{2}(\mathbb{R})}=F_{\infty, 1}^{\prime \prime}\left(\theta_{1}\right) h h \geq 0$ for any $h \in H^{2}(\mathbb{R})$ and by the non degeneracy property (Remark 2.2) we conclude that $\lambda_{0}=0$ is a simple eigenvalue whose eigenspace is spanned by $\dot{\theta}_{1}$.

To characterize the remaining part of $\sigma\left(\mathcal{L}_{\infty, 1}\right)$ we can use the min-max principle ([38], Theorem XIII.1) to conclude that

$$
\sigma\left(\mathcal{L}_{\infty, 1}\right) \cap(-\infty, 2 \underline{w}]=\left\{\lambda_{0} \leq \lambda_{1} \leq \ldots\right\}
$$

where

$$
\lambda_{j}=\sup _{V \subset H^{2}(\mathbb{R}), \operatorname{dim} V=j} \inf _{\psi \perp V,\|\psi\|=1}<\mathcal{L}_{\infty, 1} \psi, \psi>_{L^{2}(\mathbb{R})}
$$

We have that $\lambda_{1}$ is either equal to $2 \underline{w}$ or strictly less than $2 \underline{w}$ and so an eigenvalue of $\mathcal{L}_{\infty, 1}$. Since $\lambda_{0}$ is simple, we obtain that in any case $\lambda_{1}>\lambda_{0}=0$. Now, note that if $h \in H^{2}(\mathbb{R})$ is odd then $h \perp \dot{\theta}_{1}$ and hence, using e.g. the resolution of the identity relative to $\mathcal{L}_{\infty, 1}$, we obtain

$$
F_{\infty, 1}^{\prime \prime}\left(\theta_{1}\right) h h=\left\langle\mathcal{L}_{\infty, 1} h, h\right\rangle_{L^{2}(\mathbb{R})} \geq \lambda_{1}\|h\|_{L^{2}(\mathbb{R})}^{2}
$$

Then, by density, the Lemma follows.

A last consideration concerning the heteroclinic problem is about the compactness of the minimizing sequences of $F_{\infty, 1}$ with respect to the $H^{1}(\mathbb{R})$ topology. We remark that since $W$ is an even function we can restrict ourself to look for the minimizing sequences of $F_{\infty, 1}$ constituted by odd functions which are not negative for $x>0$, i.e. belonging to the space

$$
\Gamma_{\infty, 1}=\{q \in \mathcal{H} / q(x)=-q(-x) \text { and } q(x) \geq 0 \text { for } x>0\} .
$$

Then it can be proved (see e.g. Lemma 2.4 in [3]) the following result.

Lemma 2.2. (Compactness property) If $\left(q_{n}\right) \subset \Gamma_{\infty, 1}$ verifies $F_{\infty, 1}\left(q_{n}\right) \rightarrow c_{\infty, 1}$ then $\left\|q_{n}-\theta_{1}\right\|_{H^{1}(\mathbb{R})} \rightarrow 0$. In particular, for all $r>0$ there exists $\mu_{r}>0$ such that

$$
\text { if } q \in \Gamma_{\infty, 1} \text { verifies }\left\|q_{n}-\theta_{1}\right\|_{H^{1}(\mathbb{R})} \geq r \text { then } F_{\infty, 1}(q) \geq c_{\infty, 1}+\mu_{r}
$$




\subsection{The approximating one dimensional problems}

Given $L>0$, denoted $I_{L}=(-L, L)$, we consider the functional

$$
F_{L, 1}(q)=\int_{I_{L}} \frac{1}{2}|\dot{q}(x)|^{2}+W(q(x)) d x \quad \text { on } \quad \Gamma_{L, 1}=\left\{\left.q\right|_{I_{L}} / q \in \Gamma_{\infty, 1}\right\} .
$$

It is standard to show that the minima of $F_{L, 1}$ on $\Gamma_{L, 1}$ are solutions to

$$
\begin{cases}-\ddot{q}+W^{\prime}(q)=0, & x \in I_{L}, \\ q(x)=-q(-x), & x \in I_{L}, \quad\left(P_{L, 1}\right) \\ \dot{q}( \pm L)=0 & \end{cases}
$$

We set

$$
c_{L, 1}=\inf _{\Gamma_{L, 1}} F_{L, 1} \quad \text { and } \quad \mathcal{K}_{L, 1}=\left\{q \in \Gamma_{L, 1} / F_{L, 1}(q)=c_{L, 1}\right\} .
$$

The application of the direct method of the calculus of variation allows to show that $\mathcal{K}_{L, 1}$ is not empty for any $L>0$. We note that since any $q \in \mathcal{K}_{L, 1}$ is a solution to $\left(P_{L, 1}\right)$, it can be reflected about $L$ and then continued by periodicity obtaining in fact a $4 L$-periodic solution of $-\ddot{q}+W^{\prime}(q)=0$ on $\mathbb{R}$.

As described in the following Lemma the functions $q \in \mathcal{K}_{L, 1}$ are uniformly exponentially close to 1 when $x$ is large. This allows also to get informations on the asymptotic behaviour of the map $c_{L, 1}$ (see Proposition 2.2 and Lemma 2.5 in [3] for the proofs).

Lemma 2.3. (Asymptotic behaviour of $c_{L, 1}$ ). There results

i) there exists a constant $C>0$ such that

$$
0 \leq 1-q(x) \leq C e^{-\sqrt{\underline{w} / 2} x} \text { for any } x>0, L>0 \text { and } q \in \mathcal{K}_{L, 1} ;
$$

ii) the map $L>0 \rightarrow c_{L, 1}$ is monotone increasing and satisfies

$$
0 \leq c_{\infty, 1}-c_{L, 1} \leq C e^{-\sqrt{2 \underline{w}} L}, \quad \forall L>0 .
$$

The asymptotic behaviour of $c_{L, 1}$ described in (2.2) expresses a particular relation of $F_{L, 1}$ with $F_{\infty, 1}$ when $L \rightarrow+\infty$. Another related property, linked with Lemma 2.2, is the following one (see Lemma 2.6 in [3]).

Lemma 2.4. Let $L_{n} \rightarrow+\infty$ and $\left(q_{n}\right) \subset \Gamma_{L_{n}, 1}$ be such that $F_{L_{n}, 1}\left(q_{n}\right)-c_{L_{n}, 1} \rightarrow 0$ as $n \rightarrow+\infty$. Then,

$$
\left\|q_{n}-\theta_{1}\right\|_{H^{1}\left(I_{L_{n}}\right)} \rightarrow 0 \quad \text { as } n \rightarrow+\infty .
$$

By Lemma 2.4 we recover an analogous of (2.1), i.e., we have that for every $r \in(0,1)$ there exists $\mu_{r}>0$ and $\ell_{r}>0$ such that

if $L \geq \ell_{r}, q \in \Gamma_{L, 1}$ verify $\left\|q-\theta_{1}\right\|_{H^{1}\left(I_{L}\right)} \geq r$ then $F_{L, 1}(q)-c_{L} \geq \mu_{r} .(2.3)$

Consider now the space

$$
\mathcal{H}_{L}=\left\{q_{\left.\right|_{L}} / q \in \mathcal{H}\right\}
$$


which is a Hilbert space if endowed with the $H^{1}\left(I_{L}\right)$ scalar product. It is classical to show that the functional $F_{L, 1}$ is in $C^{2}\left(\mathcal{H}_{L}\right)$ and that for any $q, h, k \in \mathcal{H}_{L}$ there results

$$
F_{L, 1}^{\prime}(q) h=\int_{-L}^{L} \dot{q} \dot{h}+W^{\prime}(q) h d x \quad \text { and } \quad F_{L, 1}^{\prime \prime}(q) h k=\int_{-L}^{L} \dot{h} \dot{k}+W^{\prime \prime}(q) h k d x .
$$

For our study, it is useful to study the behaviour with respect to $L>0$ of $F_{L, 1}\left(\theta_{1}\right), F_{L, 1}^{\prime}\left(\theta_{1}\right)$ and $F_{L, 1}^{\prime \prime}\left(\theta_{1}\right)$.

First note that, thanks to the exponential asymptotic behaviour of $\theta_{1}$ (Remark 2.1) and by (1.7) we deduce the existence of a constant $C>0$ such that

$$
0<F_{\infty, 1}\left(\theta_{1}\right)-F_{L, 1}\left(\theta_{1}\right) \leq C \mathrm{e}^{-\sqrt{2 \underline{w}} L} \text { for all } L>0 .
$$

Concerning $F_{L, 1}^{\prime}\left(\theta_{1}\right)$, we note that since $\ddot{\theta}_{1}=W^{\prime}\left(\theta_{1}\right)$, integrating by parts we obtain $\left|F_{L, 1}^{\prime}\left(\theta_{1}\right) h\right|=2\left|\dot{\theta}_{1}(L) h(L)\right|$ for all $h \in \Gamma_{L, 1}$. Then, by Remark 2.1, we recover that there exists a constant $C>0$ such that

$$
\left|F_{L, 1}^{\prime}\left(\theta_{1}\right) h\right|=2\left|\dot{\theta}_{1}(L) h(L)\right| \leq C \mathrm{e}^{-\sqrt{\frac{w}{2}} L}\|h\|_{H^{1}\left(I_{L}\right)} \quad \forall L \geq 1, h \in \Gamma_{L, 1} .
$$

Finally, considering $F_{L, 1}^{\prime \prime}\left(\theta_{1}\right)$ we shall show that thanks to the stability property of $\theta_{1}$ (Lemma 2.1), if $L$ is sufficiently large then, $F_{L, 1}^{\prime \prime}\left(\theta_{1}\right)$ is a positive definite bilinear form on the space of odd functions in $H^{1}\left(I_{L}\right)$ uniformly with respect to $L$. Indeed there results

Lemma 2.5. There exists $\ell_{0}>0$ such that if $L \geq \ell_{0}, h \in H^{1}\left(I_{L}\right)$ and $h$ is odd, then

$$
F_{L, 1}^{\prime \prime}\left(\theta_{1}\right) h h \geq \frac{\lambda_{1}}{8}\|h\|_{L^{2}\left(I_{L}\right)}^{2}
$$

Proof. Set $\bar{W}=\max _{[-1,1]}\left|W^{\prime \prime}(s)\right|$ and let $h \in H^{1}\left(I_{L}\right)$ odd. If $\|\dot{h}\|_{L^{2}\left(I_{L}\right)}^{2} \geq$ $\left(\bar{W}+\frac{\lambda_{1}}{8}\right)\|h\|_{L^{2}\left(I_{L}\right)}^{2}$ we recover that

$$
F_{L, 1}^{\prime \prime}\left(\theta_{1}\right) h h \geq\|\dot{h}\|_{L^{2}\left(I_{L}\right)}^{2}-\bar{W}\|h\|_{L^{2}\left(I_{L}\right)}^{2} \geq \frac{\lambda_{1}}{8}\|h\|_{L^{2}\left(I_{L}\right)}^{2}
$$

and we have nothing more to show. So let us assume

$$
\|\dot{h}\|_{L^{2}\left(I_{L}\right)}^{2}<\left(\bar{W}+\frac{\lambda_{1}}{8}\right)\|h\|_{L^{2}\left(I_{L}\right)}^{2} .
$$

Observe that if $x_{h} \in[L / 2, L-1]$ is such that

$$
\|h\|_{H^{1}\left(x_{h}, x_{h}+1\right)}^{2}=\min _{x \in[L / 2, L-1]}\|h\|_{H^{1}(x, x+1)}^{2},
$$

then (denoting $[L / 2]$ the entire part of $L / 2$ ) there results

$$
\|h\|_{H^{1}\left(I_{L}\right)}^{2} \geq \sum_{i=0}^{[L / 2]-1}\|h\|_{H^{1}(L / 2+i, L / 2+i+1)}^{2} \geq[L / 2]\|h\|_{H^{1}\left(x_{h}, x_{h}+1\right)}^{2} .
$$

Consider the cutoff function $\beta_{h}$ equal to 1 for $|x| \leq x_{h}$, equal to 0 for $|x| \geq x_{h}+1$ and such that $\beta_{h}(x)=1-\left(|x|-x_{h}\right)$ for $0<|x|-x_{h}<1$. We have 


$$
\begin{aligned}
F_{L, 1}^{\prime \prime}\left(\theta_{1}\right) h h= & F_{L, 1}^{\prime \prime}\left(\theta_{1}\right)\left(\beta_{h} h\right)\left(\beta_{h} h\right)+2 F_{L, 1}^{\prime \prime}\left(\theta_{1}\right)\left(\left(1-\beta_{h}\right) h\right)\left(\beta_{h} h\right) \\
& +F_{L, 1}^{\prime \prime}\left(\theta_{1}\right)\left(\left(1-\beta_{h}\right) h\right)\left(\left(1-\beta_{h}\right) h\right) .
\end{aligned}
$$

By the stability property of $\theta_{1}$ (Lemma 2.1) we can estimate the first addendum as

$$
F_{L, 1}^{\prime \prime}\left(\theta_{1}\right)\left(\beta_{h} h\right)\left(\beta_{h} h\right) \geq \lambda_{1}\left\|\beta_{h} h\right\|_{L^{2}\left(I_{L}\right)}^{2} .
$$

For the third addendum, observe that, since $\theta_{1}(x) \rightarrow 1$ as $x \rightarrow+\infty$ and since $W^{\prime \prime}(1) \geq \lambda_{1}$, there exists $\ell_{0}>0$ such that $W^{\prime \prime}\left(\theta_{1}(x)\right) \geq \lambda_{1} / 2$ for any $x \geq \ell_{0} / 2$. Then if $L>\ell_{0}$, since $x_{h}>L / 2$, we recover

$$
\begin{aligned}
F_{L, 1}^{\prime \prime}\left(\theta_{1}\right)\left(\left(1-\beta_{h}\right) h\right)\left(\left(1-\beta_{h}\right) h\right) & \geq \frac{\lambda_{1}}{2}\left\|\left(1-\beta_{h}\right) h\right\|_{L^{2}(L / 2<|x|<L)}^{2} \\
& =\frac{\lambda_{1}}{2}\left\|\left(1-\beta_{h}\right) h\right\|_{L^{2}\left(I_{L}\right)}^{2} .
\end{aligned}
$$

Finally if $L>\ell_{0}$, setting $\mathcal{N}_{h}=\left(x_{h}, x_{h}+1\right)$ and using (2.7) and (2.6), we can bound from below the second addendum as follows

$$
\begin{aligned}
F_{L, 1}^{\prime \prime}( & \left.\theta_{1}\right)\left(\left(1-\beta_{h}\right) h\right)\left(\beta_{h} h\right) \\
=2 & {\left[2 \int_{\mathcal{N}_{h}} \beta_{h} \dot{h} h d x-\|h\|_{L^{2}\left(\mathcal{N}_{h}\right)}^{2}-\int_{\mathcal{N}_{h}} \dot{h} h d x\right.} \\
& \left.+\int_{\mathcal{N}_{h}}\left(1-\beta_{h}\right) \beta_{h} \dot{h}^{2} d x+\int_{\mathcal{N}_{h}}\left(1-\beta_{h}\right) \beta_{h} W^{\prime \prime}\left(\theta_{1}\right) h^{2} d x\right] \\
\geq & 4 \int_{\mathcal{N}_{h}} \beta_{h} \dot{h} h d x-2\|h\|_{L^{2}\left(\mathcal{N}_{h}\right)}^{2}-2 \int_{\mathcal{N}_{h}} \dot{h} h d x \geq-5\|h\|_{H^{1}\left(\mathcal{N}_{h}\right)}^{2} \\
\geq & -\frac{5}{[L / 2]}\|h\|_{H^{1}\left(I_{L}\right)}^{2} \geq-\frac{5}{[L / 2]}\left(1+\bar{W}+\frac{\lambda_{1}}{8}\right)\|h\|_{L^{2}\left(I_{L}\right)}^{2} .
\end{aligned}
$$

Taking $\ell_{0}$ bigger if necessary, we can assume that $\frac{5}{[L / 2]}\left(1+\bar{W}+\frac{\lambda_{1}}{8}\right) \leq \frac{\lambda_{1}}{8}$ for any $L \geq \ell_{0}$ and so, gathering the estimates above in (2.8) and using (2.6), we conclude that if $L \geq \ell_{0}$ then

$$
\begin{aligned}
F_{L, 1}^{\prime \prime}\left(\theta_{1}\right) h h & \geq \frac{\lambda_{1}}{2}\left(\left\|\beta_{h} h\right\|_{L^{2}\left(I_{L}\right)}^{2}+\left\|\left(1-\beta_{h}\right) h\right\|_{L^{2}\left(I_{L}\right)}^{2}\right)-\frac{\lambda_{1}}{8}\|h\|_{L^{2}\left(I_{L}\right)}^{2} \\
& \geq \frac{\lambda_{1}}{2}\left(\|h\|_{L^{2}\left(I_{L}\right)}^{2}-4\|h\|_{L^{2}\left(\mathcal{N}_{h}\right)}^{2}\right)-\frac{\lambda_{1}}{8}\|h\|_{L^{2}\left(I_{L}\right)}^{2} \\
& \geq \frac{\lambda_{1}}{4}\|h\|_{L^{2}\left(I_{L}\right)}^{2}-\frac{\lambda_{1}}{8}\|h\|_{L^{2}\left(I_{L}\right)}^{2}=\frac{\lambda_{1}}{8}\|h\|_{L^{2}\left(I_{L}\right)}^{2}
\end{aligned}
$$

and the Lemma follows.

Remark 2.4. By (2.3), taking the constant $\ell_{0}$ in Lemma 2.5 bigger if necessary, we can assume that setting $\tilde{W}=\frac{1}{6} \max _{s \in[-2,2]}\left|W^{\prime \prime \prime}(s)\right|$, there exists $\mu_{0}>0$ such that if $L \geq \ell_{0}, q \in \Gamma_{L, 1}$ and $F_{L, 1}(q)-c_{L, 1} \leq \mu_{0}$ then

$$
\left\|q-\theta_{1}\right\|_{H^{1}\left(I_{L}\right)} \leq \frac{\lambda_{1}}{8 \tilde{W} c_{0}}
$$

where $c_{0}$ is the immersion constant $H^{1}\left(I_{1}\right) \rightarrow L^{\infty}\left(I_{1}\right)$. 
As last result in this section we give the following Lemma which expresses a uniform coerciveness property of the functionals $F_{L}$.

Lemma 2.6. There exist a constant $C>0$, such that if $L \geq \ell_{0}, q \in \Gamma_{L, 1}$, $\|q\|_{L^{\infty}\left(I_{L}\right)} \leq 1$ are such that $F_{L, 1}(q)-c_{L, 1} \leq \mu_{0}$ then

$$
F_{L, 1}(q)-c_{L, 1} \geq \frac{\lambda_{1}}{16}\left\|q-\theta_{1}\right\|_{L^{2}\left(I_{L}\right)}^{2}-C e^{-\sqrt{\frac{w}{2}} L} .
$$

Proof. Let $L \geq \ell_{0}$ and $q \in \Gamma_{L, 1}$ such that $\|q\|_{L^{\infty}\left(I_{L}\right)} \leq 1$. We note that since $\|q\|_{L^{\infty}\left(I_{L}\right)} \leq 1$, by $(W)$ we recover that on $I_{L}$ there results

$$
\left|W(q)-W\left(\theta_{1}\right)-W^{\prime}\left(\theta_{1}\right)\left(q-\theta_{1}\right)-\frac{1}{2} W^{\prime \prime}\left(\theta_{1}(x)\right)\left(q-\theta_{1}\right)^{2}\right| \leq \frac{1}{2} \tilde{W}\left|q-\theta_{1}\right|^{3} .
$$

Since we have the identity

$$
\begin{aligned}
F_{L, 1}(q)-F_{L, 1}\left(\theta_{1}\right)= & F_{L, 1}^{\prime}\left(\theta_{1}\right)\left(q-\theta_{1}\right)+\frac{1}{2} F_{L, 1}^{\prime \prime}\left(\theta_{1}\right)\left(q-\theta_{1}\right)\left(q-\theta_{1}\right)+\int_{-L}^{L} W(q) \\
& -W\left(\theta_{1}\right)-W^{\prime}\left(\theta_{1}\right)\left(q-\theta_{1}\right)-\frac{1}{2} W^{\prime \prime}\left(\theta_{1}\right)\left(q-\theta_{1}\right)^{2} d x
\end{aligned}
$$

by (2.5), Lemma 2.5 and (2.10), we obtain that

$$
\begin{aligned}
& F_{L, 1}(q)-F_{L, 1}\left(\theta_{1}\right) \geq \frac{\lambda_{1}}{8}\left\|q-\theta_{1}\right\|_{L^{2}\left(I_{L}\right)}^{2}-C \mathrm{e}^{-\sqrt{\frac{w}{2}} L}\left\|q-\theta_{1}\right\|_{H^{1}\left(I_{L}\right)} \\
& -\frac{\tilde{W}}{2}\left\|q-\theta_{1}\right\|_{L^{3}\left(I_{L}\right)}^{3} \geq\left(\frac{\lambda_{1}}{8}-\frac{\tilde{W}}{2} c_{0}\left\|q-\theta_{1}\right\|_{H^{1}\left(I_{L}\right)}\right)\left\|q-\theta_{1}\right\|_{L^{2}\left(I_{L}\right)}^{2} \\
& -C \mathrm{e}^{-\sqrt{\frac{w}{2}} L}\left\|q-\theta_{1}\right\|_{H^{1}\left(I_{L}\right)} .
\end{aligned}
$$

Since by (2.4) and (2.2) we have that for a constant $C>0$

$$
\begin{aligned}
F_{L, 1}(q)-c_{L, 1} & =F_{L, 1}(q)-F_{L, 1}\left(\theta_{1}\right)+F_{L, 1}\left(\theta_{1}\right)-F_{\infty, 1}\left(\theta_{1}\right)+c_{\infty, 1}-c_{L, 1} \\
& \geq F_{L, 1}(q)-F_{L, 1}\left(\theta_{1}\right)-C \mathrm{e}^{-\sqrt{2 \underline{w}} L} \quad \text { for any } L>\ell_{0} \text { and } q \in \Gamma_{L, 1},
\end{aligned}
$$

by (2.11) we deduce

$$
\begin{aligned}
F_{L, 1}(q)-c_{L, 1} \geq & \left(\frac{\lambda_{1}}{8}-\frac{\tilde{W}}{2} c_{0}\left\|q-\theta_{1}\right\|_{H^{1}\left(I_{L}\right)}\right)\left\|q-\theta_{1}\right\|_{L^{2}\left(I_{L}\right)}^{2}+ \\
& -C \mathrm{e}^{-\sqrt{\frac{w}{2}} L}\left\|q-\theta_{1}\right\|_{H^{1}\left(I_{L}\right)}-C \mathrm{e}^{-\sqrt{2 \underline{w}} L} .
\end{aligned}
$$

Then the lemma follows using (2.9).

\section{The two dimensional problem}

In this section we study and characterize some variational properties of certain classes of planar entire solutions of (1.1) which are odd in each variable. Precisely in the Sect. 3.1 we recall the main steps in the construction of saddle solutions given in [3], pointing out some further variational properties. In Sect. 3.2, analogously to the study made in Sect. 2 in the one dimensional case, we consider related approximating problems studying the relationship between this class of approximating (periodic) solutions with the saddle solution. 


\subsection{Saddle Planar solutions.}

As in [3] the existence of a saddle solution of (1.1) is studied by considering the triangular set

$$
T_{\infty, 2}=\left\{(x, y) \in \mathbb{R}^{2} / x \in I_{y}, y>0\right\},
$$

and looking for minima of the renormalized functional

$$
F_{\infty, 2}(u)=\int_{0}^{+\infty} \frac{1}{2}\left\|\partial_{y} u(\cdot, y)\right\|_{L^{2}\left(I_{y}\right)}^{2}+\left(F_{y, 1}(u(\cdot, y))-c_{y, 1}\right) d y
$$

on the space

$$
\Gamma_{\infty, 2}=\left\{u \in H_{l o c}^{1}\left(T_{\infty, 2}\right) / u(\cdot, y) \in \Gamma_{y, 1} \text { for a.e. } y>0\right\} .
$$

Note that if $u \in \Gamma_{\infty, 2}$ then $u(\cdot, y) \in \Gamma_{y, 1}$ for a.e. $y>0$ and so $F_{y, 1}(u(\cdot, y))-c_{y, 1} \geq 0$ for a.e. $y>0$. Hence $F_{\infty, 2}$ is well defined on $\Gamma_{\infty, 2}$ with values in $[0,+\infty]$. The asymptotic property of the function $y \rightarrow c_{y, 1}$ described in (2.2) allows to simply show that $F_{\infty, 2}\left(\theta_{1}\right)<+\infty$ (considering $\theta_{1}$ as a function in $\Gamma_{\infty, 2}$, see Lemma 3.1 in [3]). Then we have in particular that

$$
c_{\infty, 2} \equiv \inf _{\Gamma_{\infty, 2}} F_{\infty, 2}<+\infty
$$

The functional $F_{\infty, 2}$ is weakly lower semicontinuous with respect to the $H_{l o c}^{1}\left(T_{\infty, 2}\right)$ topology and we are interested in finding minima of $F_{\infty, 2}$ on $\Gamma_{\infty, 2}$. We denote

$$
\mathcal{K}_{\infty, 2}=\left\{u \in \Gamma_{\infty, 2} \mid F_{\infty, 2}(u)=c_{\infty, 2}\right\} .
$$

Remark 3.1. From the minima of $F_{\infty, 2}$ on $\Gamma_{\infty, 2}$ we recover entire planar solutions of (1.1) recursively reflecting them with respect to the edges of $T_{\infty, 2}$. Indeed, as in Lemma 3.3 of [3], if $\bar{u} \in \mathcal{K}_{\infty, 2}$ we have

$$
\int_{T_{\infty}, 2} \nabla \bar{u} \nabla \psi+W^{\prime}(\bar{u}) \psi d x d y=0 \text { for any } \psi \in C_{0}^{\infty}\left(\mathbb{R}^{2}\right) .
$$

Then, denoting the $\pi / 2$ planar rotation matrix with $R$ and setting $T^{k}=$ $R^{k} T_{\infty, 2}$ for $k=0, \ldots, 3$, we have $\mathbb{R}^{2}=\cup_{k=0}^{3} T^{k}$, and defining

$$
\theta_{2}(x, y)=(-1)^{k} \bar{u}\left(R^{-k}(x, y)\right), \quad \forall(x, y) \in T^{k},
$$

we obtain $\theta_{2} \in H_{l o c}^{1}\left(\mathbb{R}^{2}\right)$ and, by the change of variables theorem and (3.1), $\int_{T^{k}} \nabla \theta_{2} \cdot \nabla \psi+W^{\prime}\left(\theta_{2}\right) \psi d x d y=0 \quad$ for any $\psi \in C_{0}^{\infty}\left(\mathbb{R}^{2}\right)$ and $k=0, \ldots, 3$.

Then, for any $\psi \in C_{0}^{\infty}\left(\mathbb{R}^{2}\right)$ we have

$$
\int_{\mathbb{R}^{2}} \nabla \theta_{2} \cdot \nabla \psi+W^{\prime}\left(\theta_{2}\right) \psi d x d y=\sum_{k=0}^{3} \int_{T^{k}} \nabla \theta_{2} \cdot \nabla \psi+W^{\prime}\left(\theta_{2}\right) \psi d x d y=0
$$

i.e. $\theta_{2}$ is a weak and so, by standard arguments, a classical $C^{2}\left(\mathbb{R}^{2}\right)$ solution of (1.1). We finally remark that if $\bar{u} \in \mathcal{K}_{\infty, 2}$ then $\|\bar{u}\|_{L^{\infty}\left(T_{\infty, 2}\right)} \leq 1$ (if not, defining $\bar{v}=\min \{1, \max \{-1, \bar{u}\}\}$ we have $\bar{v} \in \Gamma_{\infty, 2}$ and $\left.F_{\infty, 2}(\bar{v})<F_{\infty, 2}(\bar{u})\right)$ 
and so $\left\|\theta_{2}\right\|_{L^{\infty}\left(\mathbb{R}^{2}\right)} \leq 1$. By Schauder estimates we then obtain the existence of a constant $\bar{C}>0$ such that

$$
\|\bar{u}\|_{C^{2}\left(T_{\infty, 2}\right)}=\left\|\theta_{2}\right\|_{C^{2}\left(\mathbb{R}^{2}\right)} \leq \bar{C} \quad \text { for any } \bar{u} \in \mathcal{K}_{\infty, 2} .
$$

Given an interval $I \subset \mathbb{R}^{+}$, it is useful to consider the functional

$$
F_{I, 2}(u)=\int_{I} \frac{1}{2}\left\|\partial_{y} u(\cdot, y)\right\|_{L^{2}\left(I_{y}\right)}^{2}+\left(F_{y, 1}(u(\cdot, y))-c_{y, 1}\right) d y
$$

which is well defined on $\Gamma_{\infty, 2}$, positive and weakly lower semicontinuous with respect to the weak $H_{l o c}^{1}\left(T_{\infty, 2}\right)$ topology. The study of the existence of minima of $F_{\infty, 2}$ on $\Gamma_{\infty, 2}$ is based on the following two estimates:

i) Continuity estimate. If $u \in \Gamma_{\infty, 2}$ is such that $F_{(\sigma, \tau), 2}<+\infty$ for a certain $(\sigma, \tau) \subset(0,+\infty)$, we have that the map $y \in(\sigma, \tau) \mapsto u(\cdot, y) \in L^{2}\left(I_{\sigma}\right)$ is continuous. Indeed, if $\sigma \leq y_{1}<y_{2} \leq \tau$ then, by the Hölder inequality,

$$
\begin{aligned}
\left\|u\left(\cdot, y_{2}\right)-u\left(\cdot, y_{1}\right)\right\|_{L^{2}\left(I_{\sigma}\right)}^{2} & \leq\left(y_{2}-y_{1}\right) \int_{I_{\sigma}} \int_{y_{1}}^{y_{2}}\left|\partial_{y} u(x, y)\right|^{2} d y d x \\
& \leq\left(y_{2}-y_{1}\right) 2 F_{(\sigma, \tau), 2}(u) .
\end{aligned}
$$

ii) Coerciveness estimate. If $u \in \Gamma_{\infty, 2}$ is such that $F_{y, 1}(u(\cdot, y))-c_{y, 1} \geq$ $\mu>0$ for a.e. $y \in(\sigma, \tau) \subset \mathbb{R}^{+}$then

$$
\begin{aligned}
F_{(\sigma, \tau), 2}(u) & \geq \frac{1}{2(\tau-\sigma)}\|u(\cdot, \tau)-u(\cdot, \sigma)\|_{L^{2}\left(I_{\sigma)}\right.}^{2}+\mu(\tau-\sigma) \\
& \geq \sqrt{2 \mu}\|u(\cdot, \tau)-u(\cdot, \sigma)\|_{L^{2}\left(I_{\sigma}\right)} .
\end{aligned}
$$

Estimates (3.3) and (3.4) can be used together with (2.3) to obtain the following characterizations of asymptotic behaviour of the functions $u \in \Gamma_{\infty, 2}$ with $F_{\infty, 2}(u)<+\infty$ (see Lemma 3.2 in [3]).

Lemma 3.1. For any $r \in(0,1]$ there exists $\eta_{r}>0$ and $L_{r}>0$ such that for all $L \geq L_{r}$

$$
\text { if } u \in \Gamma_{\infty, 2} \text { and } F_{(L,+\infty), 2}(u) \leq \eta_{r} \text { then }\left\|u(\cdot, y)-\theta_{1}\right\|_{L^{2}\left(I_{y}\right)} \leq r \quad \forall y \geq L \text {. }
$$

Note that, by Lemma 3.1, we recover that if $u \in \Gamma_{\infty, 2}$ and $F_{\infty, 2}(u)<+\infty$ then

$$
\left\|u(\cdot, y)-\theta_{1}\right\|_{L^{2}\left(I_{y}\right)} \rightarrow 0 \quad \text { as } y \rightarrow+\infty .
$$

Proposition 3.1. There results $\mathcal{K}_{\infty, 2} \neq \emptyset$. Moreover, every $\bar{u} \in \mathcal{K}_{\infty, 2}$ satisfies $1>\bar{u}(x, y)>0$ for $(x, y) \in T_{\infty, 2}, x>0$ and $\left\|\bar{u}(\cdot, y)-\theta_{1}\right\|_{L^{\infty}\left(I_{y}\right)} \rightarrow 0$ as $y \rightarrow+\infty$.

Proof. It is standard to show that $\mathcal{K}_{\infty, 2} \neq \emptyset$. Indeed, letting $\left(u_{n}\right) \subset \Gamma_{2, \infty}$ be such that $F_{2, \infty}\left(u_{n}\right) \rightarrow c_{\infty, 2}$, by $(W)$, we can assume without restrictions that $\left\|u_{n}\right\|_{L^{\infty}\left(T_{\infty, 2}\right)} \leq 1$ for any $n \in \mathbb{N}$. Since for any $L>0$ there results

$$
\int_{0}^{L} \int_{-y}^{y}\left|\nabla u_{n}(x, y)\right|^{2} d x d y \leq 2\left(F_{\infty, 2}\left(u_{n}\right)+c_{L, 1} L\right)
$$


we obtain that $\left(u_{n}\right)$ is a bounded sequence in $H_{l o c}^{1}\left(T_{\infty, 2}\right)$. Hence there exists $\bar{u} \in H_{l o c}^{1}\left(T_{\infty, 2}\right)$ such that, along a subsequence, $u_{n}-\bar{u} \rightarrow 0$ weakly in $H_{l o c}^{1}\left(T_{\infty, 2}\right)$ and for a.e. $(x, y) \in T_{\infty, 2}$. By pointwise convergence, we recover that $\bar{u} \geq 0$ for $x>0$, that $\bar{u}(x, y)=-\bar{u}(-x, y)$ for a.e. $(x, y) \in T_{\infty, 2}$ and that $\|\bar{u}\|_{L^{\infty}\left(T_{\infty, 2}\right)} \leq 1$. Then $\bar{u} \in \Gamma_{\infty, 2}$ and, by semicontinuity, $F_{\infty, 2}(\bar{u})=c_{\infty, 2}$.

To show that $\bar{u}(x, y)>0$ for $x>0,(x, y) \in T_{\infty, 2}$, we note that by Remark 3.1 it holds that $-\Delta \bar{u}+W^{\prime}(\bar{u})=0$ on $T_{\infty, 2}$. Hence, since $\bar{u}(x, y) \geq 0$ for $x>0$, $(x, y) \in T_{\infty, 2}$, it satisfies the differential inequality $-\Delta \bar{u}+\max \{a(x, y), 0\} \bar{u} \geq 0$ on $T_{\infty, 2}$ when $x>0$ where

$$
a(x, y)= \begin{cases}W^{\prime}(\bar{u}) / \bar{u} & \text { if } \bar{u} \neq 0 \\ W^{\prime \prime}(0) & \text { if } \bar{u}=0\end{cases}
$$

is continuous on $T_{\infty, 2}$. Then the maximum principle tells us that either $\bar{u} \equiv 0$ or $\bar{u}>0$ on $T_{\infty, 2}$ when $x>0$. By (3.6) we conclude that $\bar{u}>0$ on $T_{\infty, 2}$ when $x>0$. Analogously one proves that $\bar{u}<1$ on $T_{\infty, 2}$ when $x>0$.

We now finally show that $\left\|\bar{u}(\cdot, y)-\theta_{1}\right\|_{L^{\infty}\left(I_{y}\right)} \rightarrow 0$ as $y \rightarrow+\infty$. For that, assume by contradiction that there exists a sequence $\left(x_{n}, y_{n}\right) \in T_{\infty, 2}$ with $y_{n} \rightarrow+\infty$ such that $\left|\bar{u}\left(x_{n}, y_{n}\right)-\theta_{1}\left(x_{n}\right)\right| \geq \rho_{0}>0$ for every $n \in \mathbb{N}$. By (3.2) we have $\|\bar{u}\|_{\mathcal{C}^{2}\left(T_{\infty, 2}\right)}<+\infty$ and so there exists $r_{0}>0$ such that $\left|\bar{u}\left(x, y_{n}\right)-\theta_{1}(x)\right| \geq \rho_{0} / 2$ whenever $\left|x-x_{n}\right| \leq r_{0},\left(x, y_{n}\right) \in T_{\infty, 2}$ and $n \in \mathbb{N}$. Then $\left\|\bar{u}\left(\cdot, y_{n}\right)-\theta_{1}\right\|_{L^{2}\left(I_{y_{n}}\right)}^{2} \geq$ $r_{0} \rho_{0}^{2} / 4$ for all $n \in \mathbb{N}$, in contradiction with (3.6).

As stated in the following Lemma the saddle solution is unique.

Lemma 3.2. There exists a unique $\bar{u} \in \mathcal{K}_{\infty, 2}$. Denoted with $\theta_{2}$ the corresponding saddle solution we have moreover $\partial_{x} \theta_{2}(x, y)>0$ and $\partial_{y} \theta_{2}(x, y)>0$ for $(x, y) \in Q_{1}=\left\{(x, y) \in \mathbb{R}^{2} / x>0, y>0\right\}$.

Proof. Let $u, v \in \mathcal{K}_{\infty, 2}$. To prove that $u=v$ we use a moving plane like argument.

As in Remark 3.1 we extend $u$ and $v$ as saddle solution on $\mathbb{R}^{2}$ of (1.1). By Proposition 3.1 we derive that $0<u(x, y), v(x, y)<1$ on $Q_{1}$ and that $u(x, y), v(x, y) \rightarrow \theta_{1}(x)$ as $y \rightarrow+\infty$ unif. w.r.t. $x \in \mathbb{R}, u(x, y), v(x, y) \rightarrow \theta_{1}(y)$ as $x \rightarrow+\infty$ unif. w.r.t. $y \in \mathbb{R}$. Then, denoting $Q_{1, r}=\{(x+r, y+r) /(x, y) \in$ $\left.Q_{1}\right\}$, we can fix $L>0$ such that $1>u(x, y), v(x, y)>1-\bar{\delta}$ on $Q_{1, L}$. Relatively to $L$ there exists $\delta_{L}>0$ such that $0<u(x, y), v(x, y)<1-\delta_{L}$ on $Q_{1} \backslash Q_{1, L}$. For $r>0$ we define $v_{r}(x, y)=v(x-r, y-r)$ for $(x, y) \in \overline{Q_{1, r}}$. By the asymptotic properties of $u$ we can fix $r_{0}>L$ such that $u>v_{r_{0}}$ on $\overline{Q_{1, r_{0}}} \backslash Q_{1, r_{0}+L}$. Moreover since $1>u(x, y), v_{r_{0}}(x, y)>1-\bar{\delta}$ on $Q_{1, r_{0}+L}$ by (1.6) we deduce that

$$
\Delta\left(u(x, y)-v_{r_{0}}(x, y)\right)^{2} \geq 2 \underline{w}\left(u(x, y)-v_{r_{0}}(x, y)\right)^{2} \quad \text { for }(x, y) \in Q_{1, r_{0}+L} .
$$

This implies that the function $u-v_{r_{0}}$ cannot have internal negative relative minima on $Q_{1, r_{0}+L}$ and so, since $u(x, y)-v_{r_{0}}(x, y)>0$ on $\partial Q_{1, r_{0}+L}$ and $\liminf u(x, y)-v_{r_{0}}(x, y) \geq 0$ as $\|(x, y)\| \rightarrow+\infty,(x, y) \in Q_{1, r_{0}+L}$, we deduce that $u(x, y)-v_{r_{0}}(x, y) \geq 0$ on $Q_{1, r_{0}+L}$ and so that $u(x, y)-v_{r_{0}}(x, y) \geq 0$ on $Q_{1, r_{0}}$. This last inequality is in fact strict since defining $a(x, y)=\left(W^{\prime}(u)-\right.$ $\left.W^{\prime}\left(v_{r_{0}}\right)\right) /\left(u-v_{r_{0}}\right)$ if $u>v_{r_{0}}$ and $a(x, y)=W^{\prime \prime}(0)$ if $u=v_{r_{0}}$, we have that $a$ is 
continuous on $Q_{1, r_{0}}$ and $-\Delta\left(u-v_{r_{0}}\right)+\max \{a(x, y), 0\}\left(u-v_{r_{0}}\right) \geq 0$ on $Q_{1, r_{0}}$. Since $u>v_{r_{0}}$ on $\overline{Q_{1, r_{0}}} \backslash Q_{1, r_{0}+L}$ by the maximum principle we conclude that $u>v_{r_{0}}$ on $\overline{Q_{1, r_{0}}}$.

We can now translate back the function $v$ considering the functions $v_{r}(x, y)=$ $v(x-r, y-r)$ on $Q_{1, r}$ for $r \in\left(0, r_{0}\right]$ showing by using again the maximum principle that $\inf \left\{r>0 / u(x, y)>v_{r}(x, y)\right.$ on $\left.Q_{1, r}\right\}=0$ and so that $u(x, y) \geq$ $v(x, y)$ on $Q_{1}$. That conclude the proof of uniqueness. For the monotonicity it is possible to use a similar argument.

Remark 3.2. The saddle solution $\theta_{2}$ enjoyes the properties:

$$
\begin{gathered}
\theta_{2}(x,-y)=-\theta_{2}(x, y), \theta_{2}(x, y)=\theta_{2}(y, x) \\
\text { on } \mathbb{R}^{2} \text { and } \theta_{2}(x, y)=\bar{u}(x, y) \text { on } T_{\infty, 2} .
\end{gathered}
$$

Since $\bar{u}(x, y)=-\bar{u}(-x, y)$ on $T_{\infty, 2}$ we then deduce that also $\theta_{2}(-x, y)=$ $-\theta_{2}(x, y)$. Then, since $\bar{u}(x, y)>0$ if $x>0$ we derive that $\theta_{2}(x, y)$ has the same sign of $x y$. Moreover, since $\left\|\bar{u}(\cdot, y)-\theta_{1}\right\|_{L^{\infty}\left(I_{y}\right)} \rightarrow 0$ as $y \rightarrow+\infty$, we finally recognize that

$$
\begin{array}{ll}
\lim _{y \rightarrow \pm \infty} \theta_{2}(x, y)= \pm \theta_{1}(x) & \text { uniformly with respect to } x \in \mathbb{R} \\
\lim _{x \rightarrow \pm \infty} \theta_{2}(x, y)= \pm \theta_{1}(y) & \text { uniformly with respect to } y \in \mathbb{R} .
\end{array}
$$

In the following Lemma we finally better characterize the convergence properties of the minimizing sequences of $F_{\infty, 2}$ on $\Gamma_{\infty, 2}$.

Lemma 3.3. If $\left(u_{n}\right) \subset \Gamma_{\infty, 2}$ verifies $\left\|u_{n}\right\|_{L^{\infty}\left(T_{\infty, 2}\right)} \leq 1$ and $F_{\infty, 2}\left(u_{n}\right) \rightarrow c_{\infty, 2}$ then $u_{n}-\theta_{2} \rightarrow 0$ in $L^{2}\left(T_{\infty, 2}\right)$.

Proof. As in the proof of Proposition 3.1, if $\left(u_{n}\right) \subset \Gamma_{\infty, 2}$ is a minimizing sequence for $F_{\infty, 2}$ such that $\left|u_{n}(x, y)\right| \leq 1$ for all $(x, y) \in T_{\infty, 2}$, we can extract a susequence (denoted again $\left(u_{n}\right)$ ) such that $u_{n} \rightarrow \theta_{2}$ weakly in $H^{1}\left(T_{L, 2}\right)$ and strongly in $L^{2}\left(T_{L, 2}\right)$ for all $L>0$, where $T_{L, 2}=\left\{(x, y) \in T_{\infty, 2} / y \in(0, L)\right\}$. The Lemma will follow once we show that

$\forall \varepsilon>0 \exists L_{\varepsilon}>0, \bar{n} \in \mathbb{N}$ such that $\left\|u_{n}-\theta_{2}\right\|_{L^{2}\left(T_{\infty, 2} \backslash T_{L, 2}\right)} \leq \varepsilon, \forall n \geq \bar{n}$.

To this aim, we first note that in general, if $\varphi, \varphi_{1}$ and $\varphi_{2}$ are lower semicontinuous functionals such that $\varphi=\varphi_{1}+\varphi_{2}$ then if $u_{n} \rightarrow u$ and $\varphi\left(u_{n}\right) \rightarrow \varphi(u)$ then also $\varphi_{1}\left(u_{n}\right) \rightarrow \varphi_{1}(u)$ and $\varphi_{2}\left(u_{n}\right) \rightarrow \varphi_{2}(u)$.

Then setting $\varphi_{1}(u)=\frac{1}{2} \int_{T_{\infty, 2}}\left|\partial_{y} u\right|^{2} d x d y+\int_{0}^{L} F_{y, 1}\left(u_{n}(\cdot, y)\right)-c_{y, 1} d y$ and $\varphi_{2}(u)=\int_{L}^{+\infty} F_{y, 1}\left(u_{n}(\cdot, y)\right)-c_{y, 1} d y$, the above remark applies to the functional $\varphi(u)=\varphi_{1}(u)+\varphi_{2}(u)=F_{\infty, 2}(u)$ and we deduce that for every $L \geq 0$

$$
\int_{L}^{+\infty} F_{y, 1}\left(u_{n}(\cdot, y)\right)-c_{y, 1} d y \rightarrow \int_{L}^{+\infty} F_{y, 1}\left(\theta_{2}(\cdot, y)\right)-c_{y, 1} d y \text { as } n \rightarrow+\infty \text {. }
$$

Let $\eta_{1}>0$ and $L_{1}>0$ be given by Lemma 3.1 in correspondence of $r=1$, let $\ell_{0}$ as defined in Lemma 2.5) and $\mu_{0}>0$ be given by Remark 2.4. 
Given $\varepsilon \in\left(0, \eta_{1}\right)$, we fix $L_{\varepsilon} \geq \max \left\{\ell_{0}, L_{1}\right\}$ such that $\int_{L_{\varepsilon}}^{+\infty} F\left(\theta_{2}(\cdot, y)\right)-m d y<$ $\frac{\varepsilon}{2}$ and we note that, by (3.8), there exists $n_{\varepsilon} \in \mathbb{N}$ such that

$$
\int_{L_{\varepsilon}}^{+\infty} F_{y, 1}\left(u_{n}(\cdot, y)\right)-c_{y, 1} d y<\varepsilon \quad \text { for all } n \geq n_{\varepsilon}
$$

We define

$$
\stackrel{\circ}{A}_{n}=\left\{y>L_{\varepsilon} / F_{y, 1}\left(u_{n}(\cdot, y)\right)-c_{y, 1}>\mu_{0}\right\},
$$

noting that, by (3.9), $\left|\AA_{n}\right| \leq \frac{\varepsilon}{\mu_{0}}$ for all $n \geq n_{\varepsilon}$. Moreover, since $\varepsilon<\eta_{1}$ and $L_{\varepsilon}>L_{1}$ by $(3.9)$ and Lemma 2.2 we recover that $\left\|u_{n}(\cdot, y)-\theta_{1}\right\|_{L^{2}\left(I_{y}\right)} \leq 1$ for any $y \geq L_{\varepsilon}$, from which we deduce

$$
\int_{\AA_{n}}\left\|u_{n}(\cdot, y)-\theta_{1}\right\|_{L^{2}\left(I_{y}\right)}^{2} d y \leq \frac{\varepsilon}{\mu_{0}} \quad \text { for any } n \geq n_{\varepsilon} .
$$

On the other hand, if $n \geq n_{\varepsilon}$ and $y \in\left(L_{\varepsilon},+\infty\right) \backslash \AA_{n}$, by definition of $\AA_{n}$ we have $F_{y, 1}\left(u_{n}(\cdot, y)\right)-c_{y, 1} \leq \mu_{0}$. Then, since $L_{\varepsilon} \geq \ell_{0}$, by Lemma 2.6 we recover that there exists a constant $C>0$, such that for any $y \geq L_{\varepsilon}, y \notin \AA_{n}$ and $n \geq n_{\varepsilon}$

$$
\left\|u_{n}(\cdot, y)-\theta_{1}\right\|_{L^{2}\left(I_{y}\right)}^{2} \leq \frac{16}{\lambda_{1}}\left(F_{y, 1}\left(u_{n}(\cdot, y)\right)-c_{y, 1}\right)+\frac{16}{\lambda_{1}} C \mathrm{e}^{-\sqrt{\frac{w}{2}} y}
$$

and so, integrating on $\left(L_{\varepsilon},+\infty\right) \backslash \AA_{n}$, by (3.9) we conclude that for any $n \geq n_{\varepsilon}$ there results

$$
\int_{\left(L_{\varepsilon},+\infty\right) \backslash \AA_{n}}\left\|u_{n}(\cdot, y)-\theta_{1}\right\|_{L^{2}\left(I_{y}\right)}^{2} d y \leq \frac{16}{\lambda_{1}} \varepsilon+\frac{16}{\lambda_{1}} C \int_{L_{\varepsilon}}^{\infty} \mathrm{e}^{-\sqrt{\frac{w}{2}} y} d y .
$$

By (3.10) and (3.11) we recover (3.7) and the Lemma follows.

\subsection{Approximating planar problem. Periodic saddle type planar solutions.}

Given $L>0$ and defined $T_{L, 2}=\left\{(x, y) \in T_{\infty, 2} / y<L\right\}$, we consider on the space $\Gamma_{L, 2}=\left\{\left.u\right|_{T_{L, 2}} / u \in \Gamma_{\infty, 2}\right\}$ the functional

$$
F_{L, 2}(u)=F_{(0, L), 2}(u)=\int_{0}^{L} \frac{1}{2}\left\|\partial_{y} u(\cdot, y)\right\|_{L^{2}\left(I_{y}\right)}^{2}+\left(F_{y, 1}(u(\cdot, y))-c_{y, 1}\right) d y
$$

We set

$$
c_{L, 2}=\inf _{\Gamma_{L, 2}} F_{L, 2} \quad \text { and } \quad \mathcal{K}_{L, 2}=\left\{u \in \Gamma_{L, 2} / F_{L, 2}(u)=c_{L, 2}\right\} .
$$

Remark 3.3. One plainly recognizes that the function $L \mapsto c_{L, 2}$ is monotone increasing for $L>0$, i.e.,

$$
\text { if } 0<L_{1}<L_{2} \text { then } c_{L_{1}, 2} \leq c_{L_{2}, 2}<c_{\infty, 2} .
$$

Moreover, as in the proof of Proposition 3.1, the application of the direct method of the calculus of variation allows to show that $\mathcal{K}_{L, 2}$ is not empty for any $L>0$ and by $(W)$ that if $u \in \mathcal{K}_{L, 2}$ then $\|u\|_{L^{\infty}\left(T_{L, 2}\right)} \leq 1$. 
Remark 3.4. Arguing as in Remark 3.1, from the minima of $F_{L, 2}$ on $\Gamma_{L, 2}$ we recover by reflections entire periodic planar solutions of (1.1). Indeed, one proves that if $u \in \mathcal{K}_{L, 2}$ then

$$
\int_{T_{L, 2}} \nabla u \nabla \psi+W^{\prime}(u) \psi d x d y=0 \quad \text { for any } \psi \in C_{0}^{\infty}\left(\mathbb{R}^{2}\right) .
$$

If $R$ is the $\pi / 2$ rotation matrix already introduced in Remark 3.1, we consider the function

$$
u_{p}(x, y)=(-1)^{k} u\left(R^{-k}(x, y)\right), \quad \forall(x, y) \in T_{L, 2}^{k},
$$

where $T_{L, 2}^{k}=R^{k} T_{L, 2}$. Setting $Q_{L}=\cup_{k=0}^{3} T_{L, 2}^{k}=(-L, L)^{2}$, by (3.12) we recognize that $\int_{Q_{L}} \nabla u \nabla \psi+W^{\prime}(u) \psi d x d y=0$ for any $\psi \in C_{0}^{\infty}\left(\mathbb{R}^{2}\right)$ and so that $u_{p}$ is a solution to $(1.1)$ on $Q_{L}=(-L, L)^{2}$ satisfying the Neumann boundary condition $\partial_{\nu} u_{p}(x, y)=0$ for $(x, y) \in \partial Q_{L}$. Then we can reflect $u_{p}$ with respect to the axes $x=L, y=L$ and then continue it by periodicity in the $x$ and $y$-direction. We then obtain a periodic entire solution of period $4 L$ both in the $x$ and $y$ variable. With abuse of notation we denote by $u_{p}$ that periodic solution. Since $\left\|u_{p}\right\|_{L^{\infty}\left(\mathbb{R}^{2}\right)} \leq 1$ for any $u \in \mathcal{K}_{L, 2}$ and $L>0$, by Schauder estimates there exists $C>0$ such that

$$
\left\|u_{p}\right\|_{C^{2}\left(\mathbb{R}^{2}\right)} \leq \bar{C} \quad \text { for any } u \in \mathcal{K}_{L, 2} \text { and } L>0 .
$$

We have now a first concentration property of the elements of $\mathcal{K}_{L, 2}$. The proof can be done adapting the argument already used in the proof of Lemma 4.2 in [7].

Lemma 3.4. For all $\varepsilon>0$ there exists $L_{\varepsilon} \geq \ell_{0}$ such that

$$
\text { if } L>L_{\varepsilon} \text { and } u \in \mathcal{K}_{L, 2} \text { then }\left\|u(\cdot, y)-\theta_{1}\right\|_{L^{\infty}\left(I_{y}\right)}<\varepsilon \quad \forall y \in\left(L_{\varepsilon}, L\right] \text {. }
$$

Remark 3.5. Since $u \in \mathcal{K}_{L, 2}$ and $\theta_{1}$ solve (1.1) on $T_{L, 2}$ we can use Lemma 3.4 to derive via the Schauder estimates that for every $\varepsilon>0$ there exists $L_{\varepsilon} \geq \ell_{0}$ such that

$$
\text { if } L>L_{\varepsilon} \text { and } u \in \mathcal{K}_{L, 2} \text { then }\left\|u(\cdot, y)-\theta_{1}\right\|_{C^{2}\left(T_{L, 2} \backslash T_{L_{\varepsilon, 2}}\right)}<\varepsilon \quad \forall y \in\left(L_{\varepsilon}, L\right] \text {. }
$$

Lemma 3.5. There exist $L_{0} \geq \ell_{0}$ and a constant $C>0$ such that for all $L \geq L_{0}$, if $u \in \mathcal{K}_{L, 2}$ then

$$
0 \leq 1-u(x, y) \leq C\left(e^{\sqrt{2 \underline{w}}\left(L_{0}-x\right)}+e^{\sqrt{2 \underline{w}}\left(L_{0}-y\right)}\right)^{1 / 2}
$$

and

$$
\left|\partial_{x} u(x, y)\right| \leq C\left(e^{\sqrt{2 \underline{w}}\left(L_{0}-x\right)}+e^{\sqrt{2 \underline{w}}\left(L_{0}-y\right)}\right)^{1 / 2} .
$$

for every $(x, y) \in T_{L, 2}$ with $x \in\left(L_{0}, L\right)$. Moreover,

$$
\forall \varepsilon>0, \exists \bar{L}_{\varepsilon}>L_{0} / \quad \text { if } u \in \mathcal{K}_{L, 2}, y \in\left(\bar{L}_{\varepsilon}, L\right) \text { then }\left\|u(\cdot, y)-\theta_{1}\right\|_{H^{1}\left(I_{y}\right)}<\varepsilon \text {. }
$$


Proof. By Remark 2.1 and (3.14), we can fix $L_{0} \geq \ell_{0}$ such that if $L>L_{0}$ and $u \in \mathcal{K}_{L, 2}$, then the corresponding periodic solution $u_{p}$ (see Remark 3.4) satisfies

$$
0 \leq 1-u_{p}(x, y) \leq \bar{\delta} \quad \text { for any }(x, y) \in \tilde{Q}_{L, L_{0}}
$$

where $\tilde{Q}_{L, L_{0}}$ is the square centered at the vertex $(L, L)$ of the triangle $T_{L, 2}$ and of side length $2\left(L-L_{0}\right)$.

Setting $\phi(x, y)=\left(1-u_{p}(x, y)\right)^{2}$ we have $0 \leq \phi(x, y) \leq \bar{\delta}^{2}$ on $\tilde{Q}_{L, L_{0}}$ and by (1.6)

$$
\Delta \phi(x, y) \geq 2 \underline{w} \phi(x, y), \quad \text { for all }(x, y) \in \tilde{Q}_{L, L_{0}}
$$

Then, by the maximum principle we obtain

$$
0 \leq 1-u_{p}(x, y) \leq \bar{\delta}\left(\frac{\cosh (\sqrt{2 \underline{w}}(x-L))+\cosh (\sqrt{2 \underline{w}}(y-L))}{\cosh \left(\sqrt{2 \underline{w}}\left(L-L_{0}\right)\right)}\right)^{1 / 2},
$$

from which (3.16) is plainly derived.

Consider now the function $\partial_{x} u_{p}$. Since it verifies $-\Delta \partial_{x} u_{p}+W^{\prime \prime}\left(u_{p}\right) \partial_{x} u_{p}=0$, we have $\Delta\left|\partial_{x} u_{p}\right|^{2} \geq W^{\prime \prime}\left(u_{p}\right)\left|\partial_{x} u_{p}\right|^{2}$ on $\mathbb{R}^{2}$. Then, by (1.6) and (3.19) we have

$$
\Delta\left|\partial_{x} u_{p}(x, y)\right|^{2} \geq 2 \underline{w}\left|\partial_{x} u_{p}(x, y)\right|^{2} \quad \text { for }(x, y) \in \tilde{Q}_{L, L_{0}} .
$$

By (3.13) and the maximum principle, we then obtain

$$
\begin{aligned}
\left|\partial_{x} u_{p}(x, y)\right| & \leq C\left(\frac{\cosh (\sqrt{2 \underline{w}}(x-L))+\cosh (\sqrt{2 \underline{w}}(y-L))}{\cosh \left(\sqrt{2 \underline{w}}\left(L-L_{0}\right)\right)}\right)^{1 / 2} \\
\quad \text { for }(x, y) & \in \tilde{Q}_{L, L_{0}}
\end{aligned}
$$

for a constant $C>0$ (independent of $L$ ) and (3.17) follows.

Finally (3.18) follows using the exponential decay property of $\theta_{1}$ described in Remark 2.1, the concentration property of $u$ displayed by (3.15), the estimates (3.16), (3.17) and the oddness with respect to the variable $x$ of $u$ and $\theta_{1}$.

Here below, using the stability property of $\theta_{1}$ given in Lemma 2.5 we give a refined version of (3.18).

Lemma 3.6. Setting $m_{0}=\frac{1}{2}\left(\frac{\lambda_{1}}{16}\right)^{1 / 2}$, there exist $\bar{L} \geq L_{0}$ and a constant $C>0$ such that if $L>\bar{L}, u \in \mathcal{K}_{L, 2}$ then

$$
\left\|u(\cdot, y)-\theta_{1}\right\|_{L^{2}\left(I_{y}\right)} \leq C e^{-m_{0} y} \text { for all } y \in[\bar{L}, L]
$$

and

$$
\left\|\partial_{x}\left(u(\cdot, y)-\theta_{1}\right)\right\|_{L^{2}\left(I_{y}\right)} \leq C e^{-m_{0} y} \text { for all } y \in[\bar{L}, L] .
$$

Proof. By $(W)$ and the $L^{\infty}$-concentration estimate (3.14) we can choose $\bar{L} \geq$ $L_{0}$ such that given $L>\bar{L}, u \in \mathcal{K}_{L, 2}$ there results that for any $y \geq \bar{L}$ and $x \in I_{y}$

$$
\left|W^{\prime}(u(x, y))-W^{\prime}\left(\theta_{1}(x)\right)-W^{\prime \prime}\left(\theta_{1}(x)\right)\left(u(x, y)-\theta_{1}(x)\right)\right| \leq \frac{\lambda_{1}}{16}\left|u(x, y)-\theta_{1}(x)\right|
$$


By (3.18) we can also assume that $\bar{L}$ is such that if $L>\bar{L}, u \in \mathcal{K}_{L, 2}$ then

$$
\left\|u(\cdot, y)-\theta_{1}\right\|_{H^{1}\left(I_{y}\right)} \leq 1 \quad \text { for every } y \in(\bar{L}, L) .
$$
define

Given $L \geq \bar{L}$ and $u \in \mathcal{K}_{L, 2}$, letting $u_{p}$ be defined as in Remark 3.4, we

$$
\phi(y)= \begin{cases}\left\|u_{p}(\cdot, y)-\theta_{1}\right\|_{L^{2}\left(I_{y}\right)}^{2} & \text { if } y \in(0 . L] \\ \left\|u_{p}(\cdot, 2 L-y)-\theta_{1}\right\|_{L^{2}\left(I_{2 L-y}\right)}^{2} & \text { if } y \in(L, 2 L)\end{cases}
$$

observing that, since $u_{p}$ is regular and symmetric with respect to $y=L$ we have that $\phi \in C^{2}((0,2 L))$ and $\phi(y)=\phi(2 L-y)$ on $(0,2 L)$. Denoted by $\langle\cdot, \cdot\rangle_{y}$ the bracket symbol for the scalar product in $L^{2}\left(I_{y}\right)$, since the functions $u, \partial_{y} u$ and $\theta_{1}$ are odd in the $x$ variable, we have that for any $y \in(0, L)$

$$
\frac{d}{d y} \phi(y)=2\left\langle u(\cdot, y)-\theta_{1}, \partial_{y} u(\cdot, y)\right\rangle_{y}=2 \int_{-y}^{y}\left(u(x, y)-\theta_{1}(x)\right) \partial_{y} u(x, y) d x .
$$

and then

$$
\ddot{\phi}(y) \geq 2\left\langle u(\cdot, y)-\theta_{1}, \partial_{y y} u(\cdot, y)\right\rangle_{y} \quad \text { for every } y \in(0, L) .
$$

Since $u$ and $\theta_{1}$ solve (1.1), by (3.22) we deduce

$$
\begin{aligned}
\langle u(\cdot, y) & \left.-\theta_{1}, \partial_{y y} u(\cdot, y)\right\rangle_{y} \geq F_{y, 1}^{\prime \prime}\left(\theta_{1}\right)\left(u(\cdot, y)-\theta_{1}\right)\left(u(\cdot, y)-\theta_{1}\right)+ \\
& -2\left(u(y, y)-\theta_{1}(y)\right) \partial_{x}\left(u(y, y)-\theta_{1}(y)\right)-\frac{\lambda_{1}}{16}\left\|u(\cdot, y)-\theta_{1}\right\|_{L^{2}\left(I_{y}\right)}^{2}
\end{aligned}
$$

for every $y \in(\bar{L}, L)$. Hence, recalling the definition of $\phi(y)$, by (3.24) and Lemma 2.5, we conclude

$\ddot{\phi}(y) \geq \frac{\lambda_{1}}{8} \phi(y)-4\left(u(y, y)-\theta_{1}(y)\right) \partial_{x}\left(u(y, y)-\theta_{1}(y)\right) \quad$ for every $y \in(\bar{L}, L)$.

Note now that by the exponential decaying of $\theta_{1}$ (Remark 2.1) and by (3.16)(3.17), we obtain that there exists $C>0$ independent of $L$ such that for $y \in(\bar{L}, L)$

$$
4\left|u(y, y)-\theta_{1}(y)\right|\left|\partial_{x}\left(u(y, y)-\theta_{1}(y)\right)\right| \leq C e^{\sqrt{2 \underline{w}}\left(L_{0}-y\right)} .
$$

Then, since $e^{\sqrt{2 \underline{w}}\left(L_{0}-y\right)} \leq \frac{\cosh (\sqrt{2 \underline{w}}(L-y))}{\cosh \left(\sqrt{2 \underline{w}}\left(L-L_{0}\right)\right)}$ on $\left(L_{0}, L\right)$, by symmetry, $(3.25)$ and (3.23) we deduce that $\phi$ verifyes

$\phi(y) \leq 1$ and $\ddot{\phi}(y) \geq \frac{\lambda_{1}}{8} \phi(y)-C \frac{\cosh (\sqrt{2 \underline{w}}(y-L))}{\cosh \left(\sqrt{2 \underline{w}}\left(L-L_{0}\right)\right)}$ for any $y \in(\bar{L}, 2 L-\bar{L})$.

Setting $\psi(y)=\frac{-C}{2 \underline{w}-\lambda_{1} / 8} \frac{\cosh (\sqrt{2 \underline{w}}(y-L))}{\cosh \left(\sqrt{2 \underline{w}}\left(L-L_{0}\right)\right)}$ (by Lemma $\left.2.1 \frac{\lambda_{1}}{8}<2 \underline{w}\right)$ and $\eta_{\alpha}(y)=\alpha \cosh \left(\sqrt{\lambda_{1} / 8}(y-L)\right)+\psi(y)-\phi(y), \quad \alpha>0, y \in[\bar{L}, 2 L-\bar{L}]$,

by (3.26) we recover that for any $\alpha>0$ there results

$$
\ddot{\eta}_{\alpha}(y)-\frac{\lambda_{1}}{8} \eta_{\alpha}(y) \leq 0 \quad \text { on }(\bar{L}, 2 L-\bar{L}) .
$$


Setting $\alpha_{0}=\frac{2}{\cosh \left(\sqrt{\lambda_{1} / 8}(\bar{L}-L)\right)}\left(\frac{C}{2 \underline{w}-\lambda_{1} / 8}+1\right)$ we have $\eta_{\alpha_{0}}(\bar{L})=\eta_{\alpha_{0}}(2 L-\bar{L})>0$ and by the maximum principle we derive that for any $y \in(\bar{L}, 2 L-\bar{L})$

$$
\begin{aligned}
\phi(y) & \leq \alpha_{0} \cosh \left(\sqrt{\lambda_{1}} 8 /(L-y)\right)+\psi(y) \\
& \leq 2\left(\frac{C}{2 \underline{w}-\lambda_{1} / 8}+1\right) \frac{\cosh \left(\sqrt{\lambda_{1} / 8}(y-L)\right)}{\cosh \left(\sqrt{\lambda_{1} / 8}(L-\bar{L})\right)} .
\end{aligned}
$$

Since on $(\bar{L}, L)$ we have $\frac{\cosh \left(\sqrt{\lambda_{1} / 8}(y-L)\right)}{\cosh \left(\sqrt{\lambda_{1} / 8}(L-\bar{L})\right)} \leq 2 \mathrm{e}^{\sqrt{\lambda_{1} / 8}(\bar{L}-y)},(3.20)$ follows.

To recover $(3.21)$, we set $\psi=u(\cdot, y)-\theta_{1}$ and integrating by parts we get

$$
\left\|\partial_{x} \psi\right\|_{L^{2}\left(I_{y}\right)}^{2} \leq 2\left|\psi(y)\left\|\partial_{x} \psi(y) \mid+(2 y)^{1 / 2}\right\| \partial_{x}^{2} \psi\left\|_{L^{\infty}\left(I_{y}\right)}\right\| \psi \|_{L^{2}\left(I_{y}\right)}\right.
$$

By (3.13) we know $\left\|\partial_{x}^{2} \psi\right\|_{L^{\infty}\left(I_{y}\right)} \leq \bar{C}$ for $y \in(\bar{L}, L)$. Using the exponential decaying of $\theta_{1}$ (Remark 2.1), (3.16), (3.17) and (3.27) we obtain

$$
2\left|\psi(y) \| \partial_{x} \psi(y)\right| \leq C e^{-\sqrt{2 \underline{w}} y} \text { and }\|\psi\|_{L^{2}\left(I_{y}\right)} \leq C \mathrm{e}^{-\frac{1}{2} \sqrt{\lambda_{1} / 8} y} \text { on }(\bar{L}, L),
$$

where $C$ is a positive constant independent on $L$. Then, taking $C$ bigger if necessary, by (3.28) we conclude

$$
\left\|\partial_{x}\left(u(\cdot, y)-\theta_{1}\right)\right\|_{L^{2}\left(I_{y}\right)}^{2} \leq C\left(e^{-\sqrt{2 \underline{w}} y}+y^{1 / 2} \mathrm{e}^{-\frac{1}{2} \sqrt{\lambda_{1} / 8} y}\right) \quad \text { on }(\bar{L}, L),
$$

from which (3.21) follows recalling that $m_{0}=\frac{1}{2} \sqrt{\lambda_{1} / 16}$.

Thanks to (3.21) we are now able to estimate the asymptotic behaviour of the function $c_{\infty, 2}-c_{L, 2}$ as $L \rightarrow+\infty$.

Lemma 3.7. There exists $C>0$ such that

$$
c_{\infty, 2}-c_{L, 2} \leq C e^{-m_{0} L} \quad \text { for all } L \geq \bar{L} .
$$

Proof. In the proof we will denote by $C$ a positive constant independent of $L$ whose value can change line to line. Letting $u \in \mathcal{K}_{L, 2}$ We consider the function $\tilde{u}(x, y)$ defined on $T_{\infty, 2}$ to be equal to $\theta_{1}(x)$ for $y \geq L+1$, equal to $u(x, y)$ for $y \leq L$ and such that for $y \in(L, L+1)$

$$
\tilde{u}(x, y)=\left\{\begin{array}{l}
(L+1-y) u(x, L)+(y-L) \theta_{1}(x) \quad-L \leq x \leq L \\
(L+1-y) u(L, L)+(y-L) \theta_{1}(x) \quad L<x<y \\
(L+1-y) u(-L, L)+(y-L) \theta_{1}(x)-y<x<-L .
\end{array}\right.
$$

We recognize that $\tilde{u} \in \Gamma_{\infty, 2}$ and so $F_{\infty, 2}(\tilde{u}) \geq c_{\infty, 2}$. Then

$$
F_{\infty, 2}(\tilde{u})=c_{L, 2}+F_{(L, L+1), 2}(\tilde{u})+\left(F_{\infty, 2}\left(\theta_{1}\right)-F_{L+1,2}\left(\theta_{1}\right)\right) \geq c_{\infty, 2}
$$

and so

$$
c_{\infty, 2}-c_{L, 2} \leq F_{(L, L+1), 2}(\tilde{u})+\left(F_{\infty, 2}\left(\theta_{1}\right)-F_{L+1,2}\left(\theta_{1}\right)\right) .
$$

Recall that by (2.4)

$$
F_{\infty, 2}\left(\theta_{1}\right)-F_{L+1,2}\left(\theta_{1}\right) \leq C \mathrm{e}^{-\sqrt{2 \underline{w}} L} \quad \text { for all } L>0 .
$$


To evaluate $F_{(L, L+1), 2}(\tilde{u})$ we recall that $F_{(L, L+1), 2}(\tilde{u})=\int_{L}^{L+1} \frac{1}{2}\left\|\partial_{y} \tilde{u}(\cdot, y)\right\|^{2}$ $L^{2}\left(I_{y}\right)+\left(F_{y, 1}(\tilde{u}(\cdot, y))-c_{y, 1}\right) d y$, and we proceed estimating the two terms $\left\|\partial_{y} \tilde{u}(\cdot, y)\right\|_{L^{2}\left(I_{y}\right)}^{2}$ and $\left(F_{y, 1}(\tilde{u}(\cdot, y))-c_{y, 1}\right)$.

By direct computation we recognize that

$$
\partial_{y} \tilde{u}(x, y)= \begin{cases}u(L, L)-\theta_{1}(x), & \text { for } y \in(L, L+1) \text { and }|x| \in(L, y) \\ u(x, L)-\theta_{1}(x), & \text { for } y \in(L, L+1) \text { and } x \in(-L, L) \\ u(-L, L)-\theta_{1}(x), & \text { for } y \in(L, L+1) \text { and } x \in(-y,-L)\end{cases}
$$

and so, since $u$ and $\theta_{1}$ are odd in the variable $x$, using Remark (2.1, (3.16) and (3.20) we obtain that for any $L \geq \bar{L}$ and $y \in(L, L+1)$,

$$
\left\|\partial_{y} \tilde{u}(\cdot, y)\right\|_{L^{2}\left(I_{y}\right)}^{2}=2\left\|u(L, L)-\theta_{1}\right\|_{L^{2}(L, y)}^{2}+\left\|u(\cdot, L)-\theta_{1}\right\|_{L^{2}\left(I_{L}\right)}^{2} \leq C \mathrm{e}^{-m_{0} L} .
$$

Considering the term $\left(F_{y, 1}(\tilde{u}(\cdot, y))-c_{y, 1}\right)$ we first note that by $(2.2)$

$$
F_{y, 1}(\tilde{u}(\cdot, y))-c_{y, 1} \leq F_{y, 1}(\tilde{u}(\cdot, y))-F_{y, 1}\left(\theta_{1}\right)+C e^{-\sqrt{2 \underline{w}} L} .
$$

Secondly we write

$F_{y, 1}(\tilde{u}(\cdot, y))-F_{y, 1}\left(\theta_{1}\right)=2\left(F_{(L, y), 1}(\tilde{u}(\cdot, y))-F_{(L, y), 1}\left(\theta_{1}\right)\right)+F_{L, 1}(\tilde{u})-F_{L, 1}\left(\theta_{1}\right)$,

and we note that for any $y \in(L, L+1)$

$$
\begin{aligned}
& F_{(L, y), 1}(\tilde{u}(\cdot, y))-F_{(L, y), 1}\left(\theta_{1}\right)=+\frac{1}{2}\left(\left\|(L-y) \dot{\theta}_{1}\right\|_{L^{2}(L, y)}^{2}-\left\|\dot{\theta}_{1}\right\|_{L^{2}(L, y)}^{2}\right) \\
& \quad+\int_{L}^{y} W\left(\theta_{1}+(L+1-y)\left(u(L, L)-\theta_{1}\right)\right) \\
& \quad-W\left(\theta_{1}\right) d x \leq\left\|\dot{\theta}_{1}\right\|_{L^{2}(L, L+1)}^{2}+C\left\|u(L, L)-\theta_{1}\right\|_{L^{2}(L, L+1)}^{2} .
\end{aligned}
$$

by Remark 2.1 and (3.16) we recover $\left\|\dot{\theta}_{1}\right\|_{L^{2}(L, L+1)}^{2} \leq C \mathrm{e}^{-\sqrt{2 \underline{w}} L}$ and $\| u(L, L)-$ $\theta_{1} \|_{L^{2}(L, L+1)}^{2} \leq C \mathrm{e}^{-\sqrt{2 \underline{w}} L}$ and so

$$
F_{(L, y), 1}(\tilde{u}(\cdot, y))-F_{(L, y), 1}\left(\theta_{1}\right) \leq C \mathrm{e}^{-\sqrt{2 \underline{w}} L} .
$$

Finally, since $\left|W\left(\theta_{1}(x)+(L+1-y)\left(u(x, L)-\theta_{1}(x)\right)\right)-W\left(\theta_{1}(x)\right)\right| \leq$ $\left|W^{\prime}\left(\theta_{1}\right)\right|\left|u(x, L)-\theta_{1}(x)\right|+C\left|u(x, L)-\theta_{1}(x)\right|^{2}$ for $x \in I_{L}$ and since $\left\|W^{\prime}\left(\theta_{1}\right)\right\|_{L^{2}\left(I_{L}\right)} \leq C$ we obtain that for any $y \in(L, L+1)$

$$
\begin{aligned}
F_{L, 1}(\tilde{u}(\cdot, y))-F_{L, 1}\left(\theta_{1}\right) \leq & \frac{1}{2}\left\|\partial_{x}\left(u(\cdot, L)-\theta_{1}\right)\right\|_{L^{2}\left(I_{L}\right)}^{2}+\left|\left\langle\dot{\theta}_{1}, \partial_{x}\left(u(\cdot, L)-\theta_{1}\right)\right\rangle_{L}\right| \\
& +\int_{-L}^{L} W\left(\theta_{1}(x)+(L+1-y)\left(u(x, L)-\theta_{1}(x)\right)\right) \\
\leq & -W\left(\theta_{1}(x)\right) d x C\left(\left\|\partial_{x}\left(u(\cdot, L)-\theta_{1}\right)\right\|_{L^{2}\left(I_{L}\right)}\right. \\
& \left.+\left\|u(\cdot, L)-\theta_{1}\right\|_{L^{2}\left(I_{L}\right)}\right)
\end{aligned}
$$

and by (3.20) and (3.21) we recover that for any $y \in(L, L+1)$

$$
F_{L, 1}(\tilde{u}(\cdot, y))-F_{L, 1}\left(\theta_{1}\right) \leq C \mathrm{e}^{-m_{0} L} .
$$

By (3.32), (3.34) and (3.35) we obtain that $F_{(L, L+1), 2}(\tilde{u}) \leq C \mathrm{e}^{-m_{0} L}$, which, together with (3.30) and (3.31), implies (3.29). 
Finally, by Lemma 2.4, adapting the argument used in [7], Lemma 4.5, we have

Lemma 3.8. Let $L_{n} \rightarrow+\infty, u_{n} \in \Gamma_{L_{n}, 2}$ such that $\left|u_{n}(x, y)\right| \leq 1$ on $T_{L_{n, 2}}$ and $F_{L_{n}, 2}\left(u_{n}\right)-c_{L_{n, 2}} \rightarrow 0$ as $n \rightarrow \infty$. Then, for any $L>0$ there results

$$
\left\|u_{n}-\theta_{2}\right\|_{L^{2}\left(T_{L, 2}\right)} \rightarrow 0 .
$$

Moreover, if $\bar{L}_{n} \in\left(0, L_{n}\right)$ is such that $\bar{L}_{n} \rightarrow+\infty$ then $F_{\left(\bar{L}_{n}, L_{n}\right), 2}\left(u_{n}\right) \rightarrow 0$.

\section{The saddle solution on $\mathbb{R}^{3}$}

In this last section we collect the results obtained above to prove Theorem 1.1. The proof iterates the variational argument already used in Sect. 2 constructing the two dimensional saddle solution $\theta_{2}$. In fact we inductively define the simplicial set

$$
T_{\infty, 3}=\left\{(x, y, z) \in \mathbb{R}^{3} / z \geq 0,(x, y) \in T_{z, 2}\right\}
$$

on which we look for a solution of (1.1) verifying the Neumann boundary condition $\left.\partial_{\nu} u\right|_{\partial T_{\infty, 3}}=0$ and the asymptotic condition $\lim _{z \rightarrow+\infty} u(x, y, z)-$ $\theta_{2}(x, y)=0$ for every $(x, y) \in T_{\infty, 2}$.

We define the space

$$
\Gamma_{\infty, 3}=\left\{u \in H_{l o c}^{1}\left(T_{\infty, 3}\right) / u(\cdot, \cdot, z) \in \Gamma_{z, 2} \text { for a.e. } z>0\right\}
$$

on which we consider the functional

$$
F_{\infty, 3}(u)=\int_{0}^{+\infty} \frac{1}{2}\left\|\partial_{z} u(\cdot, \cdot, z)\right\|_{L^{2}\left(T_{z, 2}\right)}^{2}+\left(F_{y, 2}(u(\cdot, \cdot, z))-c_{z, 2}\right) d z .
$$

Note that if $u \in \Gamma_{\infty, 3}$ then $u(\cdot, z) \in \Gamma_{z, 2}$ for a.e. $z>0$ and so $F_{y, 2}(u(\cdot, z))-$ $c_{z, 2} \geq 0$ for a.e. $z>0$. Hence $F_{\infty, 3}$ is non negative on $\Gamma_{\infty, 3}$ and, as one can simply recognize, $F_{\infty, 3}$ is weakly lower semicontinuous with respect to the $H_{l o c}^{1}\left(T_{\infty, 3}\right)$ topology. We look for a minimum of $F_{\infty, 3}$ on $\Gamma_{\infty, 3}$ and we set

$$
c_{\infty, 3}=\inf _{\Gamma_{\infty, 3}} F_{\infty, 3} \quad \text { and } \quad \mathcal{K}_{\infty, 3}=\left\{u \in \Gamma_{\infty, 3} \mid F_{\infty, 2}(u)=c_{\infty, 3}\right\} .
$$

The key Lemma to recover entire solutions of (1.1) from elements of $\mathcal{K}_{\infty, 3}$ is the following

Lemma 4.1. If $\bar{u} \in \mathcal{K}_{\infty, 3}$ then $F_{\infty, 3}(\bar{u}+\psi) \geq F_{\infty, 3}(\bar{u})$ for any $\psi \in C_{0}^{\infty}\left(\mathbb{R}^{3}\right)$. Hence

$$
\int_{T_{\infty}, 3} \nabla \bar{u} \cdot \nabla \psi+W^{\prime}(\bar{u}) \psi d x d y d z=0 \text { for any } \psi \in C_{0}^{\infty}\left(\mathbb{R}^{3}\right) .
$$

Proof. Since $W$ is even, if $\psi \in C_{0}^{\infty}\left(\mathbb{R}^{3}\right)$ satisfies $\psi(x, y, z)=-\psi(-x, y, z)$ then defined

$$
\phi(x, y, z)= \begin{cases}\bar{u}(x, y, z)+\psi(x, y, z) & \text { if } x>0 \text { and } \bar{u}(x, y, z)+\psi(x, y, z) \geq 0 \\ -\bar{u}(x, y, z)-\psi(x, y, z) & \text { if } x>0 \text { and } \bar{u}(x, y, z)+\psi(x, y, z) \leq 0 \\ -\phi(-x, y, z) & \text { if } x<0\end{cases}
$$


we have $\phi \in \Gamma_{\infty, 3}$ and $F_{\infty, 3}(\phi)=F_{\infty, 3}(\bar{u}+\psi)$. Since $\bar{u}$ is a minimum of $F_{\infty, 3}$ on $\Gamma_{\infty, 3}$, we recover that if $\psi \in C_{0}^{\infty}\left(\mathbb{R}^{3}\right)$ is such that $\psi(x, y, z)=-\psi(-x, y, z)$ then

$$
F_{\infty, 3}(\bar{u}+\psi)=F_{\infty, 3}(\phi) \geq F_{\infty, 3}(\bar{u}) .
$$

Given now any $\psi \in C_{0}^{\infty}\left(\mathbb{R}^{2}\right)$, we set $\psi_{o}(x, y, z)=\frac{1}{2}(\psi(x, y, z)-\psi(-x, y, z))$, $\psi_{e}(x, y, z)=\frac{1}{2}(\psi(x, y, z)+\psi(-x, y, z))$, and observing that the functions $\nabla \bar{u}$. $\nabla \psi_{e}$ and $\nabla \psi_{o} \cdot \nabla \psi_{e}$ are odd in the variable $x$ we recover

$$
\begin{aligned}
F_{\infty, 3}(\bar{u}+t \psi)-F_{\infty, 3}\left(\bar{u}+t \psi_{o}\right)= & \int_{T_{\infty}, 3} \frac{t^{2}}{2}\left|\nabla \psi_{e}\right|^{2}+W(\bar{u}+t \psi) \\
& -W\left(\bar{u}+t \psi_{o}\right) d x d y d z
\end{aligned}
$$

By (4.1) and since the function $W^{\prime}(\bar{u}) \psi_{e}$ is odd in the variable $x$, we conclude

$$
\begin{aligned}
& \int_{T_{\infty, 3}} \nabla \bar{u} \cdot \nabla \psi+W^{\prime}(\bar{u}) \psi d x d y d z \\
& \quad=\lim _{t \rightarrow 0^{+}} \frac{1}{t}\left(F_{\infty, 3}(\bar{u}+t \psi)-F_{\infty, 3}(\bar{u})\right) \\
& \quad \geq \lim _{t \rightarrow 0^{+}} \int_{T_{\infty, 3}} \frac{W(\bar{u}+t \psi)-W(\bar{u})}{t}+\frac{W(\bar{u})-W\left(\bar{u}+t \psi_{o}\right)}{t} d x d y d z \\
& \quad=\int_{T_{\infty}, 3} W^{\prime}(\bar{u}) \psi_{e} d x d y d z=0 .
\end{aligned}
$$

Then $\int_{T_{\infty, 3}} \nabla \bar{u} \cdot \nabla \psi+W^{\prime}(\bar{u}) \psi d x d y d z \geq 0$ for any $\psi \in C_{0}^{\infty}\left(\mathbb{R}^{3}\right)$, and the Lemma follows.

By Lemma 4.1, to construct a saddle entire solutions on $\mathbb{R}^{3}$ we can proceed recursively reflecting a function $\bar{u} \in \mathcal{K}_{\infty, 3}$. Precisely, we consider the operations of exchange of coordinates

$$
\gamma_{2,1}(x, y, z)=(y, x, z), \gamma_{3,1}(x, y, z)=(z, y, x), \gamma_{3,2}(x, y, z)=(x, z, y),
$$

to reflect with respect to the diagonal planes $\{y=x\},\{z=x\},\{z=y\}$, and the operations of changing sign of the coordinates

$$
r_{2}(x, y, z)=(x,-y, z), r_{3}(x, y, z)=(x, y,-z),
$$

to reflect with respect to the plane $\{y=0\}$ and $\{z=0\}$. Denoting

$$
\gamma_{3,0}(x, y, z)=\gamma_{2,0}(x, y, z)=(x, y, z),
$$

this operations can be composed to generate the 24 trasformations $M_{\left(k_{3}, k_{2}\right) ;\left(i_{3}, i_{2}\right)}=\gamma_{3, k_{3}} r_{3}^{i_{3}} \gamma_{2, k_{2}} r_{2}^{i_{2}}, \quad k_{3} \in\{0,1,2\}, k_{2} \in\{0,1\}\left(i_{3}, i_{2}\right) \in\{0,1\}^{2}$.

Denoting the corresponding rigid movements of $T_{\infty, 3}$

$T_{\left(k_{3}, k_{2}\right) ;\left(i_{3}, i_{2}\right)}=M_{\left(k_{3}, k_{2}\right) ;\left(i_{3}, i_{2}\right)} T_{\infty, 3}, \quad k_{3} \in\{0,1,2\}, k_{2} \in\{0,1\} \quad\left(i_{3}, i_{2}\right) \in\{0,1\}^{2}$. 


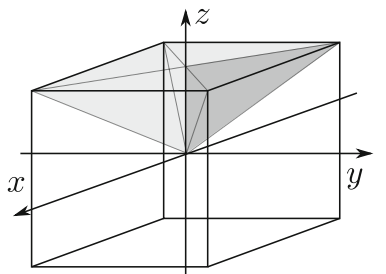

$\cup_{k_{2}=0}^{1} \cup_{i_{2}=0}^{1} T_{\left(0, k_{2}\right) ;\left(0, i_{2}\right)}$

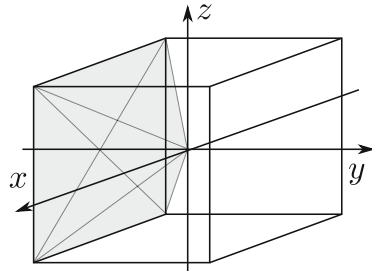

$\cup_{k_{2}=0}^{1} \cup_{i_{2}=0}^{1} T_{\left(0, k_{2}\right) ;\left(0, i_{2}\right)}$
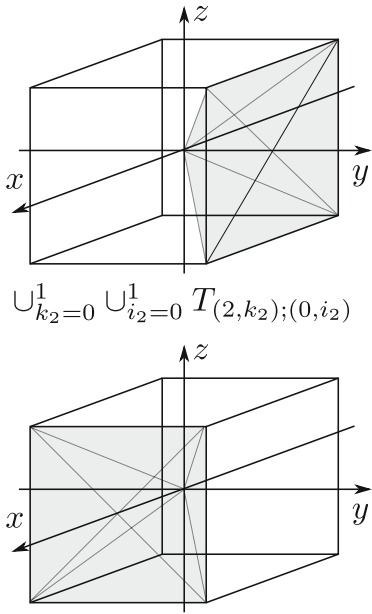

$\cup_{k_{2}=0}^{1} \cup_{i_{2}=0}^{1} T_{\left(2, k_{2}\right) ;\left(0, i_{2}\right)}$

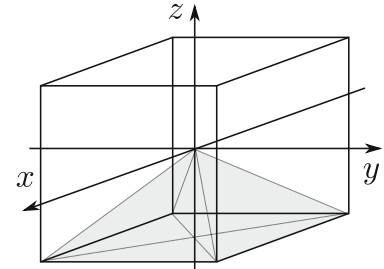

$\cup_{k_{2}=0}^{1} \cup_{i_{2}=0}^{1} T_{\left(0, k_{2}\right) ;\left(1, i_{2}\right)}$

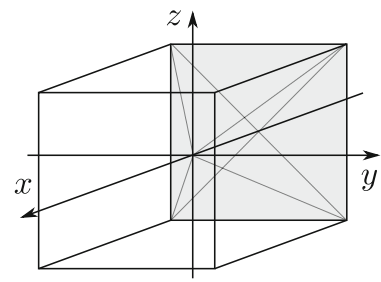

$\cup_{k_{2}=0}^{1} \cup_{i_{2}=0}^{1} T_{\left(0, k_{2}\right) ;\left(1, i_{2}\right)}$

we have $\mathbb{R}^{3}=\cup_{k_{3}=0}^{2} \cup_{k_{2}=0}^{1} \cup_{\left(i_{3}, i_{2}\right) \in\{0,1\}^{2}} T_{\left(k_{3}, k_{2}\right) ;\left(i_{3}, i_{2}\right)}$. We define

$$
\theta_{3}(x, y, z)=(-1)^{i_{2}+i_{3}} \bar{u}\left(M_{\left(k_{3}, k_{2}\right) ;\left(i_{3}, i_{2}\right)}^{-1}(x, y, z)\right), \quad \forall(x, y, z) \in T_{\left(k_{3}, k_{2}\right) ;\left(i_{3}, i_{2}\right)}
$$

noting that, since $\bar{u} \in H_{l o c}^{1}\left(T_{\infty, 3}\right)$, by the properties of the reflections operators (see e.g. [12] Lemma 9.2.) $\theta_{3} \in H_{l o c}^{1}\left(\mathbb{R}^{3}\right)$. Then, by Lemma 4.1 and by the change of variables Theorem,

$$
\begin{gathered}
\int_{T_{\left(k_{3}, k_{2}\right) ;\left(i_{3}, i_{2}\right)}} \nabla \theta_{3} \cdot \nabla \psi+W^{\prime}\left(\theta_{3}\right) \psi d x d y d z \\
=(-1)^{i_{2}+i_{3}} \int_{T_{\infty, 3}} \nabla \bar{u} \cdot \nabla \psi \circ M_{\left(k_{3}, k_{2}\right) ;\left(i_{3}, i_{2}\right)} \\
\quad+W^{\prime}(\bar{u}) \psi \circ M_{\left(k_{3}, k_{2}\right) ;\left(i_{3}, i_{2}\right)} d x d y d z=0
\end{gathered}
$$

for any $\psi \in C_{0}^{\infty}\left(\mathbb{R}^{3}\right), k_{3} \in\{0,1,2\}, k_{2} \in\{0,1\}$ and $\left(i_{3}, i_{2}\right) \in\{0,1\}^{2}$. Then

$$
\int_{\mathbb{R}^{3}} \nabla \theta_{3} \cdot \nabla \psi+W^{\prime}\left(\theta_{3}\right) \psi d x d y d z=0 \text { for any } \psi \in C_{0}^{\infty}\left(\mathbb{R}^{3}\right),
$$

i.e. $\theta_{3}$ is a weak and so, by standard bootstrap arguments, a classical $C^{2}\left(\mathbb{R}^{3}\right)$ solution of equation (1.1).

After these preliminary considerations we now proceed proving the existence of a minimum of $F_{\infty, 3}$ on $\Gamma_{\infty, 3}$.

Remark 4.1. It is simple to recognize that $c_{\infty, 3}<+\infty$ and so the minimum problem is meaningful. Indeed, considering the function $v(x, y, z)=\theta_{2}(x, y)$, we have $v \in \Gamma_{\infty, 3}$ and, by (3.29),

$F_{\infty, 3}(v)=\int_{0}^{+\infty} F_{z, 2}\left(\theta_{2}\right)-c_{z, 2} d z \leq \int_{0}^{+\infty} c_{\infty, 2}-c_{z, 2} d z \leq C \int_{0}^{+\infty} \mathrm{e}^{-m_{0} z} d z$. 
In the construction an important role is played by estimates concerning the functional $F_{\infty, 3}$ which are analogous to the continuity estimate (3.3) and the coerciveness estimate (3.4) given for the functional $F_{\infty, 2}$.

First we note that if $u \in \Gamma_{\infty, 3}$ then $F_{z, 2}(v(\cdot, \cdot, z)) \geq c_{z, 2}$ for a.e. $z>0$ and so

$$
\left\|\partial_{z} u\right\|_{L^{2}\left(T_{\infty, 3}\right)}^{2} \leq 2 F_{\infty, 3}(u) \quad \forall u \in \Gamma_{\infty, 3} .
$$

Then we observe that if $u \in \Gamma_{\infty, 3}$ we have $u(x, y, \cdot) \in H_{l o c}^{1}(z,+\infty)$ for a.e. $(x, y) \in T_{z, 2}, z>0$. Hence, if $0<z_{1}<z_{2}$, then $u\left(x, y, z_{2}\right)-u\left(x, y, z_{1}\right)=$ $\int_{z_{1}}^{z_{2}} \partial_{z} u(x, y, z) d z$ holds for all $u \in \Gamma_{\infty, 3}$ and a.e. $(x, y) \in T_{z_{1}, 2}$. So, if $u \in \Gamma_{\infty, 3}$ and $z_{1}<z_{2} \in \mathbb{R}^{+}$, by (4.2) we have

$$
\begin{aligned}
& \left\|u\left(\cdot, \cdot, z_{2}\right)-u\left(\cdot, \cdot, z_{1}\right)\right\|_{L^{2}\left(T_{z_{1}, 2}\right)}^{2} \\
& \quad=\int_{T_{z_{1}, 2}}\left|\int_{z_{1}}^{z_{2}} \partial_{z} u(x, y, z) d z\right|^{2} d x d y \\
& \quad \leq\left|z_{2}-z_{1}\right| \int_{T_{z_{1}, 2}} \int_{\mathbb{R}^{+}}\left|\partial_{z} u(x, y, z)\right|^{2} d z d x d y \leq 2 F_{\infty, 3}(u)\left|z_{2}-z_{1}\right|,
\end{aligned}
$$

which is the analogous of the continuity estimate (3.3). To recover the analogous of (3.4), given an interval $I \subset \mathbb{R}_{+}$and $u \in \Gamma_{\infty, 3}$ we set

$$
F_{I, 3}(u)=\int_{I} \frac{1}{2}\left\|\partial_{z} u(\cdot, \cdot, z)\right\|_{L^{2}\left(T_{z, 2}\right)}^{2}+\left(F_{z, 2}(u(\cdot, \cdot, z))-c_{z, 2}\right) d z .
$$

and we observe that if $u \in \Gamma_{\infty, 3}$ is such that $F_{z, 2}(u(\cdot, \cdot, z))-c_{z, 2} \geq \nu>0$ for a.e. $z \in(\sigma, \tau) \subset \mathbb{R}^{+}$, then

$$
\begin{aligned}
F_{(\sigma, \tau), 3}(u) & \geq \frac{1}{2(\tau-\sigma)}\|u(\cdot, \cdot, \tau)-u(\cdot, \cdot, \sigma)\|_{L^{2}\left(T_{2, \sigma}\right)}^{2}+\nu(\tau-\sigma) \\
& \geq \sqrt{2 \nu}\|u(\cdot, \cdot, \tau)-u(\cdot, \cdot, \sigma)\|_{L^{2}\left(T_{2, \sigma}\right)} .
\end{aligned}
$$

The estimates (4.3), (4.4) and Lemma 3.8, can be used to characterize the asymptotic behaviour, as $z \rightarrow+\infty$, of the functions $u \in \Gamma_{\infty, 3}$ such that $F_{\infty, 3}(u)<+\infty$.

Lemma 4.2. If $u \in \Gamma_{\infty, 3}$ is such that $|u(x, y, z)| \leq 1$ for a.e. $(x, y, z) \in T_{\infty, 3}$ and $F_{\infty, 3}(u)<+\infty$ then, fixed any $L>0$, we have

$$
\left\|u(\cdot, \cdot, z)-\theta_{2}\right\|_{L^{2}\left(T_{L, 2}\right)} \rightarrow 0 \quad \text { as } z \rightarrow+\infty .
$$

Proof. Let $u \in \Gamma_{\infty, 3}$ and $F_{\infty, 3}(u)<+\infty$. Since $F_{z, 2}(u(\cdot, \cdot, z))-c_{z, 2} \geq 0$ for a.e. $z>0$, there exists a sequence $z_{n} \rightarrow+\infty$ such that $F_{z_{n}, 2}\left(u\left(\cdot, \cdot, z_{n}\right)\right)-c_{z_{n}, 2} \rightarrow 0$. Fixed $L>0$, by Lemma 3.8, there exists an increasing subsequence $\left(z_{n_{k}}\right) \subset$ $\left(z_{n}\right)$ with $z_{n_{1}} \geq L$ such that $\left\|u\left(\cdot, \cdot, z_{n_{k}}\right)-\theta_{2}\right\|_{L^{2}\left(T_{L, 2}\right)} \rightarrow 0$ as $k \rightarrow+\infty$.

Assuming by contradiction that $\limsup _{z \rightarrow+\infty}\left\|u(\cdot, \cdot, z)-\theta_{2}\right\|_{L^{2}\left(T_{L, 2}\right)}=4 \delta>0$, using (4.3), we obtain the existence of a sequence of intervals $\left(\sigma_{k}, \tau_{k}\right) \subset \mathbb{R}^{+}$ such that

(i) $\sigma_{k+1}>\tau_{k} \rightarrow+\infty$,

(ii) $\left\|u\left(\cdot, \cdot, \tau_{k}\right)-u\left(\cdot, \cdot, \sigma_{k}\right)\right\|_{L^{2}\left(T_{L, 2}\right)}=\delta$,

(iii) $2 \delta \geq\left\|u(\cdot, \cdot, z)-\theta_{2}\right\|_{L^{2}\left(T_{L, 2}\right)} \geq \delta$, for every $z \in\left(\sigma_{k}, \tau_{k}\right)$. 
By Lemma 3.8, and (iii) there exist $\bar{k} \in \mathbb{N}$ and $\nu>0$ such that if $z \in \cup_{k \geq \bar{k}}\left(\sigma_{k}, \tau_{k}\right)$ then $F_{z, 2}(u(\cdot, \cdot, z))-c_{z, 2} \geq \nu$. By (4.4) and (ii) we obtain $F_{\left(\sigma_{k}, \tau_{k}\right), 3}(u) \geq \sqrt{2 \nu} \delta>0$ for all $k \geq \bar{k}$ and so, by (i), $F_{\infty, 3}(u) \geq$ $\sum_{k \geq \bar{k}} F_{\left(\sigma_{k}, \tau_{k}\right), 3}(u)=+\infty$, a contradiction.

We are now able to prove the existence of a minimum of $F_{\infty, 3}$ on $\Gamma_{\infty, 3}$.

Proposition 4.1. There exists $\bar{u} \in \Gamma_{\infty, 3}$ such that $F_{\infty, 3}(\bar{u})=c_{\infty, 3}$. Moreover for every $L>0$

$$
\left\|\bar{u}(\cdot, \cdot, z)-\theta_{2}\right\|_{L^{2}\left(T_{L, 2}\right)} \rightarrow 0 \quad \text { as } z \rightarrow+\infty .
$$

Proof. Let $\left(u_{n}\right)_{n \in \mathbb{N}} \subset \Gamma_{\infty, 3}$ be a minimizing sequence for $F_{\infty, 3}$ which we can assume such that $\left|u_{n}(x, y, z)\right| \leq 1$ for a.e. $(x, y, z) \in T_{\infty, 3}, n \in \mathbb{N}$.

It is not difficult to recognize that, fixed any $r>0$, setting $T_{r, 3}=T_{\infty, 3} \cap\{z<$ $r\}$, then $u_{n}$ is a bounded sequence on $H^{1}\left(T_{r, 3}\right)$. Indeed, since $\left|u_{n}(x, y, z)\right| \leq 1$ a.e. in $T_{\infty, 3}$ we have $\left\|u_{n}\right\|_{L^{2}\left(T_{r, 3}\right)}<+\infty$ for any $n \in \mathbb{N}$. Moreover

$$
\begin{aligned}
\| & \nabla u_{n} \|_{L^{2}\left(T_{r, 3}\right)}^{2} \\
& =\int_{0}^{r} \int_{0}^{z} \int_{-y}^{y}\left|\nabla u_{n}(x, y, z)\right|^{2} d x d y d z \\
& \leq 2 F_{\infty, 3}\left(u_{n}\right)+2 \int_{0}^{r} c_{z, 2} d z+2 \int_{0}^{r} \int_{0}^{z} c_{y, 1} d y d z \\
& \leq 2 F_{\infty, 3}\left(u_{n}\right)+2 r \tilde{c}_{\infty, 2}+r^{2} c_{\infty, 1}=2 c_{\infty, 3}+2 r c_{\infty, 2}+r^{2} c_{\infty, 1}+o(1)
\end{aligned}
$$

and our claim follows.

Thus, by a classical diagonal argument, there exists $\bar{u} \in \cap_{r>0} H^{1}\left(T_{r, 2}\right)$ and a subsequence of $\left(u_{n}\right)$, still denoted $\left(u_{n}\right)$, such that $u_{n}-\bar{u} \rightarrow 0$ weakly in $H^{1}\left(T_{r, 2}\right)$ for any $r>0$ and for a.e. $(x, y, z) \in T_{\infty, 2}$.

By pointwise convergence, we have $|\bar{u}(x, y, z)| \leq 1$ and since $u_{n} \in \Gamma_{\infty, 3}$ we derive also $\bar{u}(x, y, z)=-\bar{u}(-x, y, z)$ a.e. on $T_{\infty, 3}$ and $\bar{u}(x, y, z) \geq 0$ whenever $x \geq 0$. This proves that $\bar{u} \in \Gamma_{\infty, 3}$ and by semicontinuity we recover that $F_{\infty, 3}(\bar{u})=c_{\infty, 3}$. Finally, by Lemma 4.2 , fixed any $L>0$ we have $\| u(\cdot, \cdot, z)-$ $\theta_{2} \|_{L^{2}\left(T_{L, 2}\right)} \rightarrow 0$ as $z \rightarrow+\infty$ and the Lemma follows.

As explained above, by Proposition 4.1 and Lemma 4.1, from $\bar{u}$ we can construct a classical entire solution $\theta_{3} \in C^{2}\left(\mathbb{R}^{3}\right)$ of (1.1). Since $|\bar{u}(x, y, z)| \leq 1$ on $T_{\infty, 3}$ we have $\left|\theta_{3}(x, y, z)\right| \leq 1$ on $\mathbb{R}^{3}$. Then, by Schauder estimates, there exists a constant $\bar{C}>0$ such that

$$
\left\|\theta_{3}\right\|_{C^{2}\left(T_{\infty, 3}\right)} \leq \bar{C}
$$

This is used to finally show that $\theta_{3}$ satisfied property (iii) of Theorem 1.1.

Lemma 4.3. There results $\left\|\theta_{3}(\cdot, \cdot, z)-\theta_{2}\right\|_{L^{\infty}\left(T_{z, 2}\right)} \rightarrow 0$ as $z \rightarrow+\infty$.

Proof. Assume by contradiction that there exists $\rho_{0}>0$ and $\left(x_{n}, y_{n}, z_{n}\right) \in$ $T_{\infty, 3}$ with $z_{n} \rightarrow+\infty$ such that $\left|\theta_{3}\left(x_{n}, y_{n}, z_{n}\right)-\theta_{2}\left(x_{n}, y_{n}\right)\right| \geq 4 \rho_{0}$. By (4.5) there exists $r_{0}>0$ such that if $n \in \mathbb{N}$ and $(x, y, z) \in T_{\infty, 3}$ is such that $\left|x-x_{n}\right|,\left|y-y_{n}\right|,\left|z-z_{n}\right| \leq r_{0}$ then $\left|\theta_{3}(x, y, z)-\theta_{2}(x, y)\right| \geq 2 \rho_{0}$. Since by Proposition 4.1 we have $\left\|\theta_{3}(\cdot, \cdot, z)-\theta_{2}\right\|_{L^{2}\left(T_{L, 2}\right)} \rightarrow 0$ as $z \rightarrow+\infty$ for every 
$L>0$, we deduce that $y_{n} \rightarrow+\infty$. Since $\left\|\theta_{2}(\cdot, y)-\theta_{1}\right\|_{L^{\infty}\left(I_{y}\right)} \rightarrow 0$ as $y \rightarrow+\infty$, we recover that there exists $\bar{n}>0$ such that if $n \geq \bar{n}$ and $(x, y, z) \in T_{\infty, 3}$ is such that $\left|x-x_{n}\right|,\left|y-y_{n}\right|,\left|z-z_{n}\right| \leq r_{0}$ then $\left|\theta_{3}(x, y, z)-\theta_{1}(x)\right| \geq \rho_{0}$. In particular, if $n \geq \bar{n},\left|z-z_{n}\right| \leq r_{0}, y \in\left[y_{n}-r_{0}, y_{n}\right]$, then

$$
\left\|\theta_{3}(\cdot, y, z)-\theta_{1}\right\|_{L^{2}\left(I_{y}\right)} \geq \sqrt{2 r_{0}} \rho_{0} .
$$

By (4.6) and (2.3) we derive that there exists $\mu>0$ such that if $n \geq \bar{n}$ (with $\bar{n}$ eventually larger), $\left|z-z_{n}\right| \leq r_{0}, y \in\left[y_{n}-r_{0}, y_{n}\right]$, then

$$
F_{y, 1}\left(\theta_{3}(\cdot, y, z)\right) \geq c_{y, 1}+\mu
$$

and so in particular if $n \geq \bar{n},\left|z-z_{n}\right| \leq r_{0}$ there results

$$
F_{\left(y_{n}-r_{0}, y_{n}\right), 2}\left(\theta_{3}(\cdot, \cdot, z)\right) \geq \int_{y_{n}-r_{0}}^{y_{n}} F_{y, 1}\left(\theta_{3}(\cdot, y, z)\right)-c_{y, 1} d y \geq \mu r_{0}
$$

Denoting $A=\cup_{n \geq \bar{n}}\left[z_{n}-r_{0}, z_{n}+r_{0}\right]$, we have $\int_{A} F_{z, 2}\left(\theta_{3}(\cdot, \cdot, z)\right)-c_{z, 2} d z \leq$ $F_{\infty, 3}\left(\theta_{3}\right)<+\infty$ and so there exists a sequence $\zeta_{n} \rightarrow+\infty$ with $\zeta_{n} \in\left[z_{n}-\right.$ $\left.r_{0}, z_{n}+r_{0}\right]$, such that $F_{\zeta_{n}, 2}\left(\theta_{3}\left(\cdot, \cdot, \zeta_{n}\right)\right)-c_{\zeta_{n}, 2} \rightarrow 0$. By Lemma 3.8, since $y_{n}-r_{0} \rightarrow+\infty$ we deduce that

$$
F_{\left(y_{n}-r_{0}, y_{n}\right), 2}\left(\theta_{3}\left(\cdot, \cdot, \zeta_{n}\right)\right) \leq F_{\left(y_{n}-r_{0}, \zeta_{n}\right), 2}\left(\theta_{3}\left(\cdot, \cdot, \zeta_{n}\right)\right) \rightarrow 0,
$$

in contradiction with (4.7).

Remark 4.2. The symmetry properties of $\theta_{3}$ and Lemma 4.3 ensure that property (iii) of Theorem 1.1 is satisfied. The uniqueness of $\theta_{3}$, its positivity and monotonicity properties on the first octant are obtained again as in the proof of Lemma 3.2 by moving plane like techniques. Then Theorem 1.1 is completely proved.

\section{References}

[1] Alama, S., Bronsard, L., Gui, C.: Stationary layered solutions in $\mathbb{R}^{2}$ for an AllenCahn system with multiple well potential. Calc. Var. Partial Diff. Equ. 5, 359390 (1997)

[2] Alberti, G., Ambrosio, L., Cabré, X.: On a long-standing conjecture of E. De Giorgi: Symmetry in 3D for general nonlinearities and a local minimality property. Acta Appl. Math. 65(1-3), 9-33 (2001)

[3] Alessio, F., Calamai, A., Montecchiari, P.: Saddle-type solutions for a class of semilinear elliptic equations. Adv. Diff. Equ. 12, 361-380 (2007)

[4] Alessio, F., Montecchiari, P.: Entire solutions in $\mathbf{R}^{2}$ for a class of Allen-Cahn equations. ESAIM: COCV 11, 633-672 (2005)

[5] Alessio, F., Montecchiari, P.: Multiplicity results for a class of almost periodic Allen-Cahn type equations. Adv. Nonlinear Stud. 5, 515-549 (2005)

[6] Alessio, F., Montecchiari, P.: Brake orbits type solutions to some class of semilinear elliptic equations. Calc. Var. Partial Diff. Equ. 30, 51-83 (2007) 
[7] Alessio, F., Montecchiari, P.: Layered solutions with multiple asymptotes for non autonomous AllenCahn equations in $\mathbb{R}^{3}$. Calc. Var. PDE. doi:10.1007/ s00526-012-0495-2

[8] Ambrosio, L., Cabre, X.: Entire solutions of semilinear elliptic equations in $\mathbb{R}^{3}$ and a conjecture of De Giorgi. J. Am. Math. Soc. 13(4), 725-739 (2000)

[9] Barlow, M.T., Bass, R.F., Gui, C.: The Liouville property and a conjecture of De Giorgi. Comm. Pure Appl. Math. 53(8), 1007-1038 (2000)

[10] Berestycki, H., Caffarelli, L. A., Nirenberg, L.: Monotonicity for elliptic equations in unbounded Lipschitz domains. Comm. Pure Appl. Math. 50(11), 10891111 (1997)

[11] Berestycki, H., Hamel, F., Monneau, R.: One-dimensional symmetry for some bounded entire solutions of some elliptic equations. Duke Math. J. 103(3), 375$396(2000)$

[12] Brezis, H.: Functional Analysis, Sobolev Spaces and Partial Differential Equations. Universitext. Springer, Berlin (2011)

[13] Cabré, X., Terra, J.: Saddle-shaped solutions of bistable diffusion equations in all of $\mathbb{R}^{2 m}$. J. Eur. Math. Soc. (JEMS) 11(4), 819-943 (2009)

[14] Cabré, X., Terra, J.: Qualitative properties of saddle-shaped solutions to bistable diffusion equations. Commun. Partial Diff. Equ. 35, 1923-1957 (2010)

[15] Dang, H., Fife, P.C., Peletier, L.A.: Saddle solutions of the bistable diffusion equation. Z. Angew. Math. Phys. 43(6), 984-998 (1992)

[16] De Giorgi, E (eds.): Convergence problems for functionals and operators. In: Proceedings of International Meeting on Recent Methods in Nonlinear Analysis, Rome, (1978)

[17] del Pino, M., Kowalczyk, M., Wei, J.: A counterexample to a conjecture by De Giorgi in large dimensions. C. R. Acad. Sci. Paris, Ser. I 346, 1261-1266 (2008)

[18] del Pino, M., Kowalczyk, M., Wei, J.: On De Giorgi conjecture in dimension $N \geq 9$. Ann. Math. 174(2), 1485-1569 (2011)

[19] del Pino, M.: New entire solutions to some classical semilinear elliptic problems. In: Proceedings of the International Congress of Mathematicians ICM (2010)

[20] del Pino, M., Kowalczyk, M., Pacard, F., Wei, J.: Multiple-end solutions to the Allen-Cahn equation in $R^{2}$. J. Funct. Anal. 258(2), 458-503 (2010)

[21] del Pino, M., Kowalczyk, M., Pacard, F., Wei, J.: The Toda system and multipleend solutions of autonomous planar elliptic problems. Adv. Math. 224(4), 14621516 (2010)

[22] del Pino, M., Kowalczyk, M., Wei, J.: Entire solutions of the Allen Cahn equation and complete embedded minimal surfaces of finite total curvature. Rev. Union Math. Arg. 50(2), 95-107 (2009) 
[23] del Pino, M., Kowalczyk, M., Wei, J.: Entire solutions of the Allen-Cahn equation and complete embedded minimal surfaces. Revista de la Unión Matemática Argentina 50(2), 95-107 (2009)

[24] Farina, A.: Symmetry for solutions of semilinear elliptic equations in $\mathbb{R}^{N}$ and related conjectures. Ricerche di Matematica (in Memory of Ennio De Giorgi) 48, 129-154 (1999)

[25] Farina, A., Sciunzi, B., Valdinoci, E.: Bernstein and De Giorgi type problems: new results via a geometric approach. Ann. Sci. Norm. Super. Pisa Cl. Sci. 5, 7 (2008)

[26] Farina, A., Valdinoci, E.: The state of the art for a conjecture of de Giorgi and related problems. In: Recent progress on reaction-diffusion systems and viscosity solutions, Hackensack, NJ. World Scientific, Singapore, pp. 74-96 (2009)

[27] Farina, A., Valdinoci, E.: 1D symmetry for solutions of semilinear and quasilinear elliptic equations. Trans. Am. Math. Soc. 363, 579-609 (2011)

[28] Farina, A., Valdinoci, E.: Some results on Minimizers and stable solutions of a variational problem. Ergodic theory and dynamical systems (to appear)

[29] Ghoussoub, N., Gui, C.: On a conjecture of De Giorgi and some related problems. Math. Ann. 311, 481-491 (1998)

[30] Gui, C.: Hamiltonian identities for elliptic partial differential equations. J. Funct. Anal. 254(4), 904-933 (2008)

[31] Gui, C.: Symmetry of some entire solutions to the Allen-Cahn equation in two dimensions. J. Diff. Equ. 252(11), 5853-5874 (2012)

[32] Gui, C., Schatzman, M.: Symmetric quadruple phase transitions. Indiana Univ. Math. J. 57(2), 781-836 (2008)

[33] Kowalczyk, M., Liu, Y.: Nondegeneracy of the saddle solution of the Allen-Cahn equation. Proc. AMS 139(1), 4319-4329 (2011)

[34] Kowalczyk, M., Liu, Y., Pacard, F.: The space of 4-ended solutions to the AllenCahn equation on the plane. Ann. IHP Analise Nonlinearire, 29(5), 761-781 (2012)

[35] Kowalczyk, M., Liu, Y., Pacard, F.: The Classification of Four end solutions to the Allen-Cahn equation on the plane (2011) (preprint)

[36] Rabinowitz, P.H.: Solutions of heteroclinic type for some classes of semilinear elliptic partial differential equations. J. Math. Sci. Univ. Tokio 1, 525-550 (1994)

[37] Rabinowitz, P.H., Stredulinsky, E.: Extensions of Moser Bangert theory: locally minimal solutions. In: Progress in Nonlinear Differential Equations and Their applications, vol. 81, Birkhäuser, Basel (2011)

[38] Reed, M., Simon, B.: Methods of Modern Mathematical Physics IV: Analysis of Operators. Academic Press, New York (1978) 
[39] Savin, O.: Phase transition: regularity of flat level sets. Ann. Math. 169, 4778 (2009)

[40] Savin, O., Sciunzi, B., Valdinoci, E.: Flat level set regularity of p-Laplace phase transitions. In: Memoirs of the American Mathematical Society, vol. 182 (2006)

[41] Schatzman, M.: On the stability of the saddle solution of Allen-Cahn's equation. Proc. R. Soc. Edinburgh Sect. A 125(6), 1241-1275 (1995)

Francesca Alessio and Piero Montecchiari

Dipartimento di Ingegneria Industriale e Scienze Matematiche

Università Politecnica delle Marche

Via Brecce Bianche

60131 Ancona

Italy

e-mail: alessio@dipmat.univpm.it

Piero Montecchiari

e-mail: p.montecchiari@univpm.it

Received: 15 June 2012.

Accepted: 4 November 2012. 